\title{
QUANTUM ELECTRODYNAMICS IN A LASER AND THE ELECTRON LASER COLLISION
}

\author{
ZHANG QI-REN \\ School of Physics, Peking University, Beijing 100871, China
}

\begin{abstract}
Quantum electrodynamics in a laser is formulated, in which the electron-laser interaction is exactly considered, while the interaction of an electron and a single photon is considered by perturbation. The formulation is applied to the electron-laser collisions. The effect of coherence between photons in the laser is therefore fully considered in these collisions. The possibility of $\gamma$-ray laser generation by use of this kind of collision is discussed.

Key words: Quantum electrodynamics in a laser, Electron-laser collisions, Distortion of electron wave by the laser, $\gamma$-ray laser generation.

PACS number(s):12.20.-m, 11.80.-m, 42.55.Ah, 42.50.Ct, 42.55.Vc
\end{abstract}

\section{INTRODUCTION}

The matter-laser interaction is an interesting topic in physics. Many interesting new phenomena are waiting us to explore [1 10]. Theoretically, the problem is how to solve the wave equation of charged particles under the joint actions of the laser and of other origins. Therefore, the easiest problem in this area is the electron-laser collision. To solve it one needs only to solve the wave equation of an electron in a classical electromagnetic field presenting the laser only. For a plane wave laser it is already solved [11, 12]. Of course, even in a simple electron-laser collision, some photons other than those in the source laser may be created or absorbed. The fundamental theory for researching these processes has to be quantum electrodynamics.

Quantum electrodynamics is the most successful theory in physics [13]. Its main applications were to various processes participated by a few photons and electrons only. In these applications, it is called the quantum electrodynamics in vacuum. Its results always agree with observations to a precision as high as achievable by the present experiments. This is partially because the smallness of the fundamental electromagnetic coupling constant, and the renormalizability of the theory, so that the perturbation theory is applicable. However, in the electron-laser processes, the interaction, though is still of the electromagnetic origin, is not weak because the coherence of huge number photons interacting with the electron. To handle the non-perturbation character in these processes one needs some new methods in quantum electrodynamics. Here we formulate a quantum electrodynamics in laser, in which the vacuum state of electromagnetic field in usual formulation is substituted by a coherent state of photons describing the laser. In the following we will show that, this formulation is a new picture of quantum electrodynamics, equivalent to its other pictures, but specially suitable for the treatment of electromagnetic processes with a laser. The interaction of electrons with the laser is exactly considered, but the interaction between electrons and the photons other than those in the laser is still handled by perturbation. The electron laser scattering is demonstrated as an ex- ample, with special interest in the possible construction of a $\gamma$-ray laser.

In section [I] we reform the quantum electrodynamics, so that instead to quantize the electromagnetic field itself we quantize the fluctuation of it around one of its classical solutions, the laser field. As a contrast, the usual quantization method of electrodynamics in vacuum is also shortly reviewed. In section [III we apply the obtained formulation to the case of quantum electrodynamics in a circularly polarized laser, and transform it into a picture, in which the Hamiltonian is divided into two parts, one is time independent and another is a small perturbation. In section IV we solve the eigen-equation of the time independent unperturbed Hamiltonian, obtain a complete orthonormal set of eigenfunctions for the electron. By expanding the electron field in terms of this set of eigenfunctions, we quantize the electron field in the laser. The formulation is completed. In section $\mathrm{V}$ we apply this formulation to the electron laser collision, the expression of the cross section is obtained, and some examples of numerical results are shown. In section VI more numerical results are shown. Especially, the case of large $\mathcal{N}$ is discussed in some details. $\mathcal{N}$ is an integer, may be imagined to be the number of photons in the laser, which participate the collision. But from an analysis of the mathematical derivation we see, its presence is a result of the electron wave distortion by the laser. In section VII the possibility of making a $\gamma$-ray laser by the electron laser collision is considered. Section VIII is for discussions.

\section{QUANTIZATION OF ELECTROMAGNETIC FIELD AROUND ONE OF ITS CLASSICAL EXPRESSION, THE QUANTUM ELECTRODYNAMICS IN A LASER}

Consider an electrodynamic system composed of electrons and photons. In the nature unit system $(c=\hbar=$ 1 ), its Lagrangian density is

$$
\mathcal{L}=-\bar{\Psi}\left[\gamma_{\mu}\left(\partial_{\mu}+\mathrm{i} e A_{\mu}\right)+m\right] \Psi-\frac{1}{4} F_{\mu \nu} F_{\mu \nu}
$$


with

$$
F_{\mu \nu}=\partial_{\mu} A_{\nu}-\partial_{\nu} A_{\mu} .
$$

$\Psi$ denotes the electron field, $\left[A_{\mu}\right]$ with $\mu=1,2,3,4$ denote the 4-electromagnetic potential, $e$ is the absolute value of the electron charge, and $m$ is the electron mass. Notations in Lurie's book[14] are used here. By a standard procedure collecting the contributions of longitudinal and temporal components of the electromagnetic potential into a coulomb energy

$$
H_{c}=\frac{1}{2} \int \mathrm{d}^{3} x \int \mathrm{d}^{3} x^{\prime} \Psi^{\dagger}(\boldsymbol{x}) \Psi(\boldsymbol{x}) \frac{\alpha}{\left|\boldsymbol{x}-\boldsymbol{x}^{\prime}\right|} \Psi^{\dagger}\left(\boldsymbol{x}^{\prime}\right) \Psi\left(\boldsymbol{x}^{\prime}\right)
$$

one obtains the Hamiltonian

$$
H=H_{1}+H_{2}+H_{c}
$$

of the system in Coulomb gauge, with

$$
\begin{aligned}
& H_{1}=\int \Psi^{\dagger}[\boldsymbol{\alpha} \cdot(\boldsymbol{p}+e \boldsymbol{A})+\beta m] \Psi \mathrm{d}^{3} x, \\
& H_{2}=\frac{1}{2} \int\left(\mathcal{E}^{2}+\mathcal{H}^{2}\right) \mathrm{d}^{3} x
\end{aligned}
$$

$\alpha \equiv e^{2} / 4 \pi=1 / 137 . \cdots$ is the fine structure constant showing the strength of the electromagnetic interaction; $\boldsymbol{p}=-\mathrm{i} \boldsymbol{\nabla}$ is the momentum of an electron; $\mathcal{E}=-\partial \boldsymbol{A} / \partial t$ is the transverse electric field strength under the Coulomb gauge condition

$$
\nabla \cdot A=0
$$

$\mathcal{H}=\boldsymbol{\nabla} \times \boldsymbol{A}$ is the magnetic field strength. Since $H_{c}$ is of the higher order of the small parameter $\sqrt{\alpha}$, and is proportional to the square of electron number density, one needs to consider the first two terms only in eq.(4) for processes participated by a few electrons and photons, unless the electron number density in the problem is as high as that in atoms. It is to consider the Hamiltonian

$$
H_{s}=H_{1}+H_{2} .
$$

Expand $\Psi(\boldsymbol{x})$ and $\bar{\Psi}(\boldsymbol{x})$ in terms of the complete orthonormal set of eigenfunctions $\frac{1}{\sqrt{(2 \pi)^{3}}} u_{\sigma}(\boldsymbol{p}) \exp (\mathrm{i} \boldsymbol{p} \cdot \boldsymbol{x})$ and $\frac{1}{\sqrt{(2 \pi)^{3}}} v_{\sigma}(\boldsymbol{p}) \exp (-\mathrm{i} \boldsymbol{p} \cdot \boldsymbol{x})$ of the single electron energy operator $\boldsymbol{\alpha} \cdot(-\mathrm{i} \nabla)+\beta m$ in vacuum, in which bispinors $u_{\sigma}(\boldsymbol{p})$ and $v_{\sigma}(\boldsymbol{p})$ satisfy equations

$$
\begin{aligned}
& (\boldsymbol{\alpha} \cdot \boldsymbol{p}+\beta m) u_{\sigma}(\boldsymbol{p})=E(p) u_{\sigma}(\boldsymbol{p}), \\
& (\boldsymbol{\alpha} \cdot \boldsymbol{p}-\beta m) v_{\sigma}(\boldsymbol{p})=E(p) v_{\sigma}(\boldsymbol{p}),
\end{aligned}
$$

with

$$
\begin{aligned}
& E(p)=\sqrt{p^{2}+m^{2}}, \\
& u_{\sigma}^{\dagger}(\boldsymbol{p}) u_{\sigma^{\prime}}(\boldsymbol{p})=\delta_{\sigma \sigma^{\prime}} \\
& v_{\sigma}^{\dagger}(\boldsymbol{p}) v_{\sigma^{\prime}}(\boldsymbol{p})=\delta_{\sigma \sigma^{\prime}}, \\
& u_{\sigma}^{\dagger}(\boldsymbol{p}) v_{\sigma^{\prime}}(\boldsymbol{p})=v_{\sigma}^{\dagger}(\boldsymbol{p}) u_{\sigma^{\prime}}(\boldsymbol{p})=0,
\end{aligned}
$$

$\sigma$ is the spin index. We have

$$
\begin{aligned}
\Psi(\boldsymbol{x}) & =\int \frac{\mathrm{d}^{3} p}{\sqrt{(2 \pi)^{3}}} \sum_{\sigma}\left[c_{\sigma}(\boldsymbol{p}) u_{\sigma}(\boldsymbol{p}) \mathrm{e}^{\mathrm{i} \boldsymbol{p} \cdot \boldsymbol{x}}\right. \\
& \left.+d_{\sigma}^{\dagger}(\boldsymbol{p}) v_{\sigma}(\boldsymbol{p}) \mathrm{e}^{-\mathrm{i} \boldsymbol{p} \cdot \boldsymbol{x}}\right], \\
\bar{\Psi}(\boldsymbol{x}) & =\int \frac{\mathrm{d}^{3} p}{\sqrt{(2 \pi)^{3}}} \sum_{\sigma}\left[d_{\sigma}(\boldsymbol{p}) \bar{v}_{\sigma}(\boldsymbol{p}) \mathrm{e}^{\mathrm{i} \boldsymbol{p} \cdot \boldsymbol{x}}\right. \\
& \left.+c_{\sigma}^{\dagger}(\boldsymbol{p}) \bar{u}_{\sigma}(\boldsymbol{p}) \mathrm{e}^{-\mathrm{i} \boldsymbol{p} \cdot \boldsymbol{x}}\right] .
\end{aligned}
$$

In the same way, expanding $\boldsymbol{A}(\boldsymbol{x})$ in terms of transverse plane waves $\frac{1}{\sqrt{(2 \pi)^{3} 2 k}} \boldsymbol{e}_{i} \mathrm{e}^{\mathrm{i} \boldsymbol{k} \cdot \boldsymbol{x}}, i=1,2$, with

$$
\begin{aligned}
\boldsymbol{e}_{i}^{*} \cdot \boldsymbol{e}_{i^{\prime}} & =\delta_{i i^{\prime}} \\
\boldsymbol{e}_{i}^{*} \cdot \boldsymbol{k} & =0
\end{aligned}
$$

we have

$$
\boldsymbol{A}(\boldsymbol{x})=\int \mathrm{d}^{3} k \sum_{i}\left[a_{i}(\boldsymbol{k}) \frac{\boldsymbol{e}_{i} \mathrm{e}^{\mathrm{i} \boldsymbol{k} \cdot \boldsymbol{x}}}{\sqrt{(2 \pi)^{3} 2 k}}+a_{i}^{\dagger}(\boldsymbol{k}) \frac{\boldsymbol{e}_{i}^{*} \mathrm{e}^{-\mathrm{i} \boldsymbol{k} \cdot \boldsymbol{x}}}{\sqrt{(2 \pi)^{3} 2 k}}\right]
$$

$c_{\sigma}, c_{\sigma}^{\dagger}, d_{\sigma}, d_{\sigma}^{\dagger}, a_{i}$ and $a_{i}^{\dagger}$ are expansion coefficients. Quantization rules are

$$
\begin{aligned}
& c_{\sigma}(\boldsymbol{p}) c_{\sigma^{\prime}}\left(\boldsymbol{p}^{\prime}\right)+c_{\sigma^{\prime}}\left(\boldsymbol{p}^{\prime}\right) c_{\sigma}(\boldsymbol{p}) \\
&= d_{\sigma}(\boldsymbol{p}) d_{\sigma^{\prime}}\left(\boldsymbol{p}^{\prime}\right)+d_{\sigma^{\prime}}\left(\boldsymbol{p}^{\prime}\right) d_{\sigma}(\boldsymbol{p}) \\
&= c_{\sigma}(\boldsymbol{p}) d_{\sigma^{\prime}}\left(\boldsymbol{p}^{\prime}\right)+d_{\sigma^{\prime}}\left(\boldsymbol{p}^{\prime}\right) c_{\sigma}(\boldsymbol{p}) \\
&= c_{\sigma}(\boldsymbol{p}) d_{\sigma^{\prime}}^{\dagger}\left(\boldsymbol{p}^{\prime}\right)+d_{\sigma^{\prime}}^{\dagger}\left(\boldsymbol{p}^{\prime}\right) c_{\sigma}(\boldsymbol{p})=0, \\
& c_{\sigma}(\boldsymbol{p}) c_{\sigma^{\prime}}^{\dagger}\left(\boldsymbol{p}^{\prime}\right)+c_{\sigma^{\prime}}^{\dagger}\left(\boldsymbol{p}^{\prime}\right) c_{\sigma}(\boldsymbol{p}) \\
&= d_{\sigma}(\boldsymbol{p}) d_{\sigma^{\prime}}^{\dagger}\left(\boldsymbol{p}^{\prime}\right)+d_{\sigma^{\prime}}^{\dagger}\left(\boldsymbol{p}^{\prime}\right) d_{\sigma}(\boldsymbol{p}) \\
&= \delta_{\sigma \sigma^{\prime}} \delta\left(\boldsymbol{p}-\boldsymbol{p}^{\prime}\right) \\
& a_{i}(\boldsymbol{k}) a_{i^{\prime}}\left(\boldsymbol{k}^{\prime}\right)-a_{i^{\prime}}\left(\boldsymbol{k}^{\prime}\right) a_{i}(\boldsymbol{k})=0 \\
& a_{i}(\boldsymbol{k}) a_{i^{\prime}}^{\dagger}\left(\boldsymbol{k}^{\prime}\right)-a_{i^{\prime}}^{\dagger}\left(\boldsymbol{k}^{\prime}\right) a_{i}(\boldsymbol{k})=\delta_{i i^{\prime}} \delta\left(\boldsymbol{k}-\boldsymbol{k}^{\prime}\right) .
\end{aligned}
$$

$a_{i}(\boldsymbol{k})$ and $a_{i}^{\dagger}(\boldsymbol{k})$ always commute with $c_{\sigma}(\boldsymbol{p})$ and $d_{\sigma}(\boldsymbol{p})$. The vacuum state $|0\rangle$ is defined by

$$
c_{\sigma}(\boldsymbol{p})|0\rangle=d_{\sigma}(\boldsymbol{p})|0\rangle=a_{i}(\boldsymbol{k})|0\rangle=0 .
$$

It is a state without any electron, positron or photon, and is therefore a vacuum state in its usual meaning. This formulation is called the quantum electrodynamics in vacuum.

In the Schrödinger picture, operators $c_{\sigma}, d_{\sigma}$ and $a_{i}$ are time independent, and the state vector $|t\rangle$ depends on time $t$ according to the Schrödinger equation

$$
\mathrm{i} \frac{\mathrm{d}|t\rangle}{\mathrm{d} t}=H_{s}|t\rangle \text {. }
$$

Consider a time dependent unitary transformation generated by the operator $\mathrm{e}^{\mathrm{i} H_{2} t}$. It transforms the state vector 1) and the operator $O$ into

$$
|\rangle_{(e i)} \equiv \mathrm{e}^{\mathrm{i} H_{2} t}|\rangle \quad \text { and } \quad O_{(e i)} \equiv \mathrm{e}^{\mathrm{i} H_{2} t} O \mathrm{e}^{-\mathrm{i} H_{2} t}
$$


respectively. Substituting (19) into (6) we have

$$
H_{2}=\int \sum_{i} \frac{k}{2}\left(a_{i}^{\dagger}(\boldsymbol{k}) a_{i}(\boldsymbol{k})+a_{i}(\boldsymbol{k}) a_{i}^{\dagger}(\boldsymbol{k})\right) \mathrm{d}^{3} k .
$$

It shows $c_{\sigma(e i)}(\boldsymbol{p})=c_{\sigma}(\boldsymbol{p}), d_{\sigma(e i)}(\boldsymbol{p})=d_{\sigma}(\boldsymbol{p})$, but $a_{i(e i)}(\boldsymbol{k})=a_{i}(\boldsymbol{k}) \mathrm{e}^{-\mathrm{i} k t}$. The free motion of the electromagnetic field is therefore already considered in the transformation. In this picture, the time dependence of the state is governed by the equation

$$
\mathrm{i} \frac{\mathrm{d}|t\rangle_{(e i)}}{\mathrm{d} t}=H_{1(e i)}|t\rangle_{(e i)}
$$

$H_{1(e i)}$ is the Hamiltonian for the electron motion and the electron-photon interaction in this picture, with

$$
\begin{aligned}
\boldsymbol{A}_{(e i)}(\boldsymbol{x}) & =\int \mathrm{d}^{3} k \sum_{i}\left[a_{i}(\boldsymbol{k}) \frac{\boldsymbol{e}_{i} \mathrm{e}^{\mathrm{i}(\boldsymbol{k} \cdot \boldsymbol{x}-k t)}}{\sqrt{(2 \pi)^{3} 2 k}}\right. \\
& \left.+a_{i}^{\dagger}(\boldsymbol{k}) \frac{\boldsymbol{e}_{i}^{*} \mathrm{e}^{-\mathrm{i}(\boldsymbol{k} \cdot \boldsymbol{x}-k t)}}{\sqrt{(2 \pi)^{3} 2 k}}\right] .
\end{aligned}
$$

We may therefore call this picture the electroninteraction picture, and denote it by the subscript $(e i)$.

A laser is a classical limit of the intense electromagnetic wave and is well described by the classical vector potential

$$
\begin{aligned}
\boldsymbol{A}_{c}(\boldsymbol{x}) & =\int \mathrm{d}^{3} k \sum_{i}\left[a_{i c}(\boldsymbol{k}) \frac{\boldsymbol{e}_{i} \mathrm{e}^{\mathrm{i}(\boldsymbol{k} \cdot \boldsymbol{x}-k t)}}{\sqrt{(2 \pi)^{3} 2 k}}\right. \\
& \left.+a_{i c}^{*}(\boldsymbol{k}) \frac{\boldsymbol{e}_{i}^{*} \mathrm{e}^{-\mathrm{i}(\boldsymbol{k} \cdot \boldsymbol{x}-k t)}}{\sqrt{(2 \pi)^{3} 2 k}}\right]
\end{aligned}
$$

$a_{i c}$ and $a_{i c}^{*}$ are c-number expansion coefficients. Now, instead $\boldsymbol{A}(\boldsymbol{x})$, let us consider the fluctuation

$$
\begin{aligned}
\boldsymbol{A}^{\prime}(\boldsymbol{x}) & \equiv \boldsymbol{A}_{(e i)}(\boldsymbol{x})-\boldsymbol{A}_{c}(\boldsymbol{x})=\int \mathrm{d}^{3} k \sum_{i}\left[a_{i}^{\prime}(\boldsymbol{k}) \frac{\boldsymbol{e}_{i} \mathrm{e}^{\mathrm{i}(\boldsymbol{k} \cdot \boldsymbol{x}-k t)}}{\sqrt{(2 \pi)^{3} 2 k}}\right. \\
& \left.+a_{i}^{\prime \dagger}(\boldsymbol{k}) \frac{\boldsymbol{e}_{i}^{*} \mathrm{e}^{-\mathrm{i}(\boldsymbol{k} \cdot \boldsymbol{x}-k t)}}{\sqrt{(2 \pi)^{3} 2 k}}\right]
\end{aligned}
$$

of vector potential $\boldsymbol{A}$ around the laser $\boldsymbol{A}_{c}$, in which

$$
a_{i}^{\prime}(\boldsymbol{k})=a_{i}(\boldsymbol{k})-a_{i c}(\boldsymbol{k}) .
$$

Since $a_{i c}(\boldsymbol{k})$ and $a_{i c}^{*}(\boldsymbol{k})$ are c-numbers, we see

$$
\begin{aligned}
& a_{i}^{\prime}(\boldsymbol{k}) a_{i^{\prime}}^{\prime}\left(\boldsymbol{k}^{\prime}\right)-a_{i^{\prime}}^{\prime}\left(\boldsymbol{k}^{\prime}\right) a_{i}^{\prime}(\boldsymbol{k})=0, \\
& a_{i}^{\prime}(\boldsymbol{k}) a_{i^{\prime}}^{\prime \dagger}\left(\boldsymbol{k}^{\prime}\right)-a_{i^{\prime}}^{\prime \dagger}\left(\boldsymbol{k}^{\prime}\right) a_{i}^{\prime}(\boldsymbol{k})=\delta_{i i^{\prime}} \delta\left(\boldsymbol{k}-\boldsymbol{k}^{\prime}\right) .
\end{aligned}
$$

These equations may also be viewed as the quantization rules for the electromagnetic field, equivalent to equations (22) and (23). This is the quantization of electromagnetic field around one of its classical process. The 'vacuum state' $\left|\left[a_{i^{\prime} c}\left(\boldsymbol{k}^{\prime}\right)\right] ; 0\right\rangle$ of electromagnetic field fluctuation in this formulation is defined by

$$
a_{i}^{\prime}(\boldsymbol{k})\left|\left[a_{i^{\prime} c}\left(\boldsymbol{k}^{\prime}\right)\right] ; 0\right\rangle=0 .
$$

By (32), we see

$$
a_{i}(\boldsymbol{k})\left|\left[a_{i^{\prime} c}\left(\boldsymbol{k}^{\prime}\right)\right] ; 0\right\rangle=a_{i c}(\boldsymbol{k})\left|\left[a_{i^{\prime} c}\left(\boldsymbol{k}^{\prime}\right)\right] ; 0\right\rangle .
$$

It shows that this is a coherent state of electromagnetic field with amplitudes $\left[a_{i^{\prime} c}\left(\boldsymbol{k}^{\prime}\right)\right]$, describing the laser (30). We call this formulation the quantum electrodynamics in a laser.

\section{QUANTUM ELECTRODYNAMICS IN A CIRCULARLY POLARIZED LASER, THE ROTATION PICTURE}

In the Coulomb gauge, a circularly polarized laser is well described by the classical vector potential

$$
\boldsymbol{A}_{c}(\boldsymbol{x})=A_{c}\left\{\boldsymbol{x}_{0} \cos \left[k_{c}(z-t)\right]+\boldsymbol{y}_{0} \sin \left[k_{c}(z-t)\right]\right\} .
$$

It is a plane wave circularly polarized in the $x-y$ plane and propagating along the $z$ direction, with a wave vector $\boldsymbol{k}_{c}=k_{c} \boldsymbol{z}_{0}$ and an amplitude $A_{c} . \boldsymbol{x}_{0}, \boldsymbol{y}_{0}$ and $\boldsymbol{z}_{0}$ are unit vectors along $x, y$ and $z$ directions respectively. Writing it in the form (30), we see

$$
a_{i c}(\boldsymbol{k})=\sqrt{(2 \pi)^{3} k_{c}} A_{c} \delta_{i 1} \delta\left(\boldsymbol{k}-\boldsymbol{k}_{c}\right)
$$

and

$$
\boldsymbol{e}_{1} \equiv \boldsymbol{e}=\frac{\boldsymbol{x}_{0}-\mathrm{i} \boldsymbol{y}_{0}}{\sqrt{2}} \quad\left(\text { for } \boldsymbol{k}=\boldsymbol{k}_{c}\right)
$$

By equations (5), (26) and (31) we obtain

$$
\begin{aligned}
H_{1(e i)} & =H_{0}+H^{\prime}, \\
H_{0} & =\int \Psi^{\dagger}\left[\boldsymbol{\alpha} \cdot\left(\boldsymbol{p}+e \boldsymbol{A}_{c}\right)+\beta m\right] \Psi \mathrm{d}^{3} x, \\
H^{\prime} & =e \int \Psi^{\dagger} \boldsymbol{\alpha} \cdot \boldsymbol{A}^{\prime} \Psi \mathrm{d}^{3} x .
\end{aligned}
$$

Equation (37) shows, $H_{0}$ is time dependent. Fortunately, a time dependent unitary transformation generated by the operator $\mathrm{e}^{-\mathrm{i} k_{c} t J_{z}}$ may remove the time dependence of $H_{0}$, in which

$$
J_{z} \equiv \int \Psi^{\dagger} j_{z} \Psi \mathrm{d}^{3} x
$$

is the $z$-component of the angular momentum for the electron system, with

$$
j_{z}=-\mathrm{i} \frac{\partial}{\partial \varphi}+\frac{\Sigma_{z}}{2}
$$

being the $z$-component of the angular momentum for one electron. $\varphi$ is the azimuth angle of the electron and $\Sigma_{z}$ 
is the $z$-component of the Pauli matrices. This transformation changes the state vector |\rangle and operator $O$ into

$$
|\rangle_{r}=\mathrm{e}^{-\mathrm{i} k_{c} t J_{z}}|\rangle \text { and } O_{r}=\mathrm{e}^{-\mathrm{i} k_{c} t J_{z}} O \mathrm{e}^{\mathrm{i} k_{c} t J_{z}}
$$

respectively. It is a rotation around the $z$-direction with angular velocity $k_{c}$, the resulting picture is therefore called the rotation picture, and denoted by the subscript $r$. Simple derivation shows

$$
H_{0 r}=\int \Psi^{\dagger}\left\{\boldsymbol{\alpha} \cdot \boldsymbol{p}+e A_{c}\left[\alpha_{x} \cos \left(k_{c} z\right)+\alpha_{y} \sin \left(k_{c} z\right)\right]+\beta m\right\} \Psi \mathrm{d}^{3} x,
$$

which is indeed time independent. The time dependence of state vector in this picture is governed by

$$
\mathrm{i} \frac{\partial|\rangle_{r}}{\partial t}=\left(k_{c} J_{z}+H_{0 r}+H_{r}^{\prime}\right)|\rangle_{r} .
$$

Take

$$
\begin{aligned}
H_{0 r+} & \equiv H_{0 r}+k_{c} J_{z}=\int \Psi^{\dagger}\left\{\boldsymbol{\alpha} \cdot \boldsymbol{p}+e A_{c}\left[\alpha_{x} \cos \left(k_{c} z\right)\right.\right. \\
& \left.\left.+\alpha_{y} \sin \left(k_{c} z\right)\right]+\beta m+k_{c} j_{z}\right\} \Psi \mathrm{d}^{3} x
\end{aligned}
$$

to be the unperturbed Hamiltonian, and

$$
\begin{aligned}
& H^{\prime}{ }_{r}=e \int \mathrm{d}^{3} x \int \mathrm{d}^{3} k \sum_{i} \Psi^{\dagger}\left[a_{i}^{\prime}(\boldsymbol{k}) \frac{\boldsymbol{\alpha} \cdot \boldsymbol{e}_{i r} \mathrm{e}^{\mathrm{i}\left(\boldsymbol{k}_{r} \cdot \boldsymbol{x}-k t\right)}}{\sqrt{(2 \pi)^{3} 2 k}}\right. \\
& \left.+a_{i}^{\prime \dagger}(\boldsymbol{k}) \frac{\boldsymbol{\alpha} \cdot \boldsymbol{e}_{i r}^{*} \mathrm{e}^{-\mathrm{i}\left(\boldsymbol{k}_{r} \cdot \boldsymbol{x}-k t\right)}}{\sqrt{(2 \pi)^{3} 2 k}}\right] \Psi
\end{aligned}
$$

to be the perturbation, with

$$
\begin{aligned}
\boldsymbol{k}_{r} & =\left[k_{x} \cos \left(k_{c} t\right)-k_{y} \sin \left(k_{c} t\right)\right] \boldsymbol{x}_{0} \\
& +\left[k_{x} \sin \left(k_{c} t\right)+k_{y} \cos \left(k_{c} t\right)\right] \boldsymbol{y}_{0}+k_{z} \boldsymbol{z}_{0}, \\
\boldsymbol{e}_{i r} & =\left[e_{i x} \cos \left(k_{c} t\right)-e_{i y} \sin \left(k_{c} t\right)\right] \boldsymbol{x}_{0} \\
& +\left[e_{i x} \sin \left(k_{c} t\right)+e_{i y} \cos \left(k_{c} t\right)\right] \boldsymbol{y}_{0}+e_{i z} \boldsymbol{z}_{0},
\end{aligned}
$$

one may solve the equation (47) by the usual perturbation procedure. The smallness of electromagnetic coupling constant $\sqrt{\alpha}$ makes the procedure reliable.

From eqs. (50) and (51) we see that plane waves $\boldsymbol{e}_{i r} \mathrm{e}^{\mathrm{i}\left(\boldsymbol{k}_{r} \cdot \boldsymbol{x}-k t\right)}$ in the interaction Hamiltonian (49) rotate around the $\mathrm{z}$ axis with angular velocity $k_{c}$. The picture transformation (45) stops the rotation of the circularly polarized laser environment in $H_{0}$ and starts the rotation of the quantum modes of the electromagnetic field fluctuations around an opposite direction in $H^{\prime}$. However, the contents of the theory and the resulting prediction of observation do not change.

\section{QUANTIZATION OF THE ELECTRON FIELD IN A CIRCULARLY POLARIZED LASER AND THE ROTATION-INTERACTION PICTURE}

To quantize the electron field $\Psi$ in the rotation picture, one has to expand it in terms of a set of orthonormal functions $\left[\psi_{n}\right]$, which diagonalizes $H_{0 r+}$. It requires

$$
\begin{aligned}
& \left\{\boldsymbol{\alpha} \cdot(-\mathrm{i} \boldsymbol{\nabla})+e A_{c}\left[\alpha_{x} \cos \left(k_{c} z\right)+\alpha_{y} \sin \left(k_{c} z\right)\right]\right. \\
& \left.+\beta m+k_{c} j_{z}\right\} \psi_{n}(\boldsymbol{x})=\varepsilon_{n} \psi_{n}(\boldsymbol{x}) .
\end{aligned}
$$

This is an eigenequation, $\psi_{n}$ is the eigenfunction and $\varepsilon_{n}$ is the eigenvalue. We derive the eigenfunctions from the Wolkow solution [11, 12] of the Dirac equation for an electron in a plane electromagnetic wave. In the circularly polarized plane wave (37), the Dirac equation is

$$
\mathrm{i} \frac{\partial \psi}{\partial t}=\left\{\boldsymbol{\alpha} \cdot(-\mathrm{i} \boldsymbol{\nabla})+e A_{c}\left[\alpha_{x} \cos \phi+\alpha_{y} \sin \phi\right]+\beta m\right\} \psi
$$

with $\phi \equiv k_{c}(z-t)$. Its Wolkow solution is

$$
\psi_{w}(x)=\mathrm{e}^{\mathrm{i} p_{\mu} x_{\mu}} \mathcal{F}(\phi),
$$

with

$$
\begin{aligned}
& \mathcal{F}(\phi)=\left\{1-e A_{c} k_{c}\left[\alpha_{x} \cos \phi+\alpha_{y} \sin \phi\right.\right. \\
& \left.\left.+\mathrm{i}\left(\Sigma_{y} \cos \phi-\Sigma_{x} \sin \phi\right)\right] / 2 k_{\mu} p_{\mu}\right\} \exp \left\{-\mathrm{i} e\left[2 A _ { c } \left(p_{x} \sin \phi\right.\right.\right. \\
& \left.\left.\left.-p_{y} \cos \phi\right)+e A_{c}^{2} \phi+C\right] / 2 k_{\mu} p_{\mu}\right\} u,
\end{aligned}
$$

$C$ is a constant, $u$ is a Dirac bispinor satisfying the Dirac equation

$$
\left(\gamma_{\mu} p_{\mu}-\mathrm{i} m\right) u=0
$$

for a free electron. The solution is characterized by quantum numbers $p_{\mu}, \mu=1,2,3,4$, satisfying the energymomentum relation $p_{\mu} p_{\mu}=-m^{2}$ of a free electron. Write $p_{x}=p_{\perp} \cos \varphi_{p}$ and $p_{y}=p_{\perp} \sin \varphi_{p}$, we see

$$
\begin{aligned}
\psi_{r}(\boldsymbol{x}, t) & \equiv \mathrm{e}^{-\mathrm{i} k_{c} t J_{z}} \psi_{w}(\boldsymbol{x}, t)=\mathrm{e}^{\mathrm{i}\left(p_{z} z-E t\right)}\left\{1-\frac{e A_{c}}{2\left(p_{z}-E\right)}\left[\alpha_{x} \cos k_{c} z+\alpha_{y} \sin k_{c} z+\mathrm{i}\left(\Sigma_{y} \cos k_{c} z-\Sigma_{x} \sin k_{c} z\right)\right]\right\} \\
& \times \exp \left\{\mathrm{i} p_{\perp}\left[\left(x-\frac{e A_{c}}{k_{c}\left(p_{z}-E\right)} \sin k_{c} z\right) \cos \left(k_{c} t+\varphi_{p}\right)+\left(y+\frac{e A_{c}}{k_{c}\left(p_{z}-E\right)} \cos k_{c} z\right) \sin \left(k_{c} t+\varphi_{p}\right)\right]\right\} \\
& \times \exp \left[-\mathrm{i} \frac{e^{2} A_{c}^{2}(z-t)}{2\left(p_{z}-E\right)}-\mathrm{i} C^{\prime}\right] \mathrm{e}^{-\mathrm{i} \frac{k_{c} t \Sigma_{z}}{2}} u .
\end{aligned}
$$


$C^{\prime}$ is another constant, $E= \pm \sqrt{\boldsymbol{p}^{2}+m^{2}}$ is the energy of a free electron with momentum $\boldsymbol{p}$. Solving (56) one has

$$
\begin{gathered}
u=u_{+}+u_{-} \mathrm{e}^{\mathrm{i} \sigma \varphi_{p}}, \\
u_{+}=\sqrt{\frac{E+m}{2 E}}\left[\begin{array}{c}
1 \\
\frac{p_{z} \sigma}{E+m}
\end{array}\right] \chi_{\sigma}, \quad u_{-}=\sqrt{\frac{E+m}{2 E}}\left[\begin{array}{c}
0 \\
\frac{p_{\perp}}{E+m}
\end{array}\right] \chi_{-\sigma},
\end{gathered}
$$

$\sigma= \pm 1$, and $\chi_{ \pm \sigma}$ is an eigen-spinor of $\Sigma_{z}$ with eigenvalue $\pm \sigma$.Write

$$
x=\rho \cos \varphi, \quad y=\rho \sin \varphi,
$$

$$
\left.\begin{array}{rl}
x^{\prime} & =x-\frac{e A_{c}}{k_{c}\left(p_{z}-E\right)} \sin k_{c} z=\rho^{\prime} \cos \varphi^{\prime}, \\
y^{\prime} & =y+\frac{e A_{c}}{k_{c}\left(p_{z}-E\right)} \cos k_{c} z=\rho^{\prime} \sin \varphi^{\prime}
\end{array}\right\} .
$$

They define $\rho, \varphi, x^{\prime}, y^{\prime}, \rho^{\prime}$ and $\varphi^{\prime}$. It is a $z$-dependent coordinate transformation from $x, y$ to $\rho^{\prime}, \varphi^{\prime}$.Using the formula

$$
\mathrm{e}^{\mathrm{i} a \cos \theta}=\sum_{n=-\infty}^{\infty} \mathrm{i}^{n} \mathrm{~J}_{n}(a) \mathrm{e}^{\mathrm{i} n \theta}
$$

in which $\mathrm{J}_{n}(a)$ is a Bessel function of order $n$ in variable $a$, we find

$$
\begin{aligned}
\psi_{r}(\boldsymbol{x}, t) & =\mathrm{e}^{\mathrm{i}\left(p_{z} z-E t\right)}\left\{1-\frac{e A_{c}}{2\left(p_{z}-E\right)}\left[\alpha_{x} \cos k_{c} z+\alpha_{y} \sin k_{c} z+\mathrm{i}\left(\Sigma_{y} \cos k_{c} z-\Sigma_{x} \sin k_{c} z\right)\right]\right\} \\
& \times \exp \left\{\mathrm{i}\left[p_{\perp} \rho^{\prime} \cos \left(k_{c} t+\varphi_{p}-\varphi^{\prime}\right)-\frac{e^{2} A_{c}^{2}(z-t)}{2\left(p_{z}-E\right)}-C^{\prime}\right]\right\}\left[\mathrm{e}^{-\mathrm{i} \frac{\sigma}{2} k_{c} t} u_{+}+\mathrm{e}^{\mathrm{i} \frac{\sigma}{2}\left(k_{c} t+2 \varphi_{p}\right)} u_{-}\right] \\
& =\mathrm{e}^{\mathrm{i}\left(p_{z} z-E t+\frac{\sigma}{2} \varphi_{p}\right)}\left\{1-\frac{e A_{c}}{2\left(p_{z}-E\right)}\left[\alpha_{x} \cos k_{c} z+\alpha_{y} \sin k_{c} z+\mathrm{i}\left(\Sigma_{y} \cos k_{c} z-\Sigma_{x} \sin k_{c} z\right)\right]\right\} \\
& \times \exp \left\{-\mathrm{i}\left[\frac{e^{2} A_{c}^{2}(z-t)}{2\left(p_{z}-E\right)}+C^{\prime}\right]\right\} \sum_{n=-\infty}^{\infty} \mathrm{i}^{n} \mathrm{~J}_{n}\left(p_{\perp} \rho^{\prime}\right) \mathrm{e}^{-\mathrm{i} n \varphi^{\prime}}\left[\mathrm{e}^{\mathrm{i}\left(n-\frac{\sigma}{2}\right)\left(k_{c} t+\varphi_{p}\right)} u_{+}+\mathrm{e}^{\mathrm{i}\left(n+\frac{\sigma}{2}\right)\left(k_{c} t+\varphi_{p}\right)} u_{-}\right] \\
& =\sum_{n=-\infty}^{\infty} \mathrm{e}^{\mathrm{i}\left(n \varphi_{p}-C^{\prime}\right)} \mathrm{e}^{-\mathrm{i} \varepsilon_{n} t} U_{n}(\boldsymbol{x})
\end{aligned}
$$

with

$$
\begin{gathered}
\varepsilon_{n}=E+\frac{e^{2} A_{c}^{2}}{2\left(E-p_{z}\right)}+\left(\frac{\sigma}{2}-n\right) k_{c}, \\
U_{n}(\boldsymbol{x})=\exp \left\{\mathrm{i}\left[p_{z}+\frac{e^{2} A_{c}^{2}}{2\left(E-p_{z}\right)}\right] z\right\} \\
\times\left\{1-\frac{e A_{c}}{2\left(p_{z}-E\right)}\left[\alpha_{x} \cos k_{c} z+\alpha_{y} \sin k_{c} z+\mathrm{i}\left(\Sigma_{y} \cos k_{c} z-\Sigma_{x} \sin k_{c} z\right)\right]\right\} \\
\times\left[\mathrm{i}^{n} \mathrm{~J}_{n}\left(p_{\perp} \rho^{\prime}\right) \mathrm{e}^{-\mathrm{i} n \varphi^{\prime}} \mathrm{P}_{+}+\mathrm{i}^{n-\sigma} \mathrm{J}_{n-\sigma}\left(p_{\perp} \rho^{\prime}\right) \mathrm{e}^{-\mathrm{i}(n-\sigma) \varphi^{\prime}} \mathrm{P}_{-}\right] u_{\sigma}(0),
\end{gathered}
$$

in which $\mathrm{P}_{ \pm}=\frac{1 \pm \sigma \Sigma_{z}}{2}$ is the projection operator, $u_{\sigma}(0)=$ $u_{+}+u_{-}$is $u$ for $\varphi_{p}=0$. Since $\psi_{w}$ is a solution of the Dirac equation (53), $\psi_{r}$ defined in (57) is a solution of the equation

$$
\begin{aligned}
\mathrm{i} \frac{\partial \psi}{\partial t} & =\left\{\boldsymbol{\alpha} \cdot(-\mathrm{i} \boldsymbol{\nabla})+e A_{c}\left[\alpha_{x} \cos \left(k_{c} z\right)+\alpha_{y} \sin \left(k_{c} z\right)\right]\right. \\
& \left.+\beta m+k_{c} j_{z}\right\} \psi
\end{aligned}
$$

Substituting the last expression of the equation (63) into this equation, we see

$$
\begin{aligned}
& \sum_{n=-\infty}^{\infty} \mathrm{e}^{\mathrm{i}\left(n \varphi_{p}-C^{\prime}\right)} \mathrm{e}^{-\mathrm{i} \varepsilon_{n} t}\left\{\boldsymbol{\alpha} \cdot(-\mathrm{i} \boldsymbol{\nabla})+e A_{c}\left[\alpha_{x} \cos \left(k_{c} z\right)\right.\right. \\
& \left.\left.+\alpha_{y} \sin \left(k_{c} z\right)\right]+\beta m+k_{c} j_{z}-\varepsilon_{n}\right\} U_{n}(\boldsymbol{x})=0 .
\end{aligned}
$$

For given $\boldsymbol{p}, E$ and $\sigma, \varepsilon_{n}$ is a monotonic function of $n$. Functions $\mathrm{e}^{-\mathrm{i} \varepsilon_{n} t}$ are linearly independent of each other for different $n$. The necessary and sufficient condition for the validity of equation (67) is

$$
\begin{aligned}
& \left\{\boldsymbol{\alpha} \cdot(-\mathrm{i} \boldsymbol{\nabla})+e A_{c}\left[\alpha_{x} \cos \left(k_{c} z\right)+\alpha_{y} \sin \left(k_{c} z\right)\right]\right. \\
& \left.+\beta m+k_{c} j_{z}\right\} U_{n}(\boldsymbol{x})=\varepsilon_{n} U_{n}(\boldsymbol{x}) .
\end{aligned}
$$


Comparing with equation (52) we see $\psi_{n}(\boldsymbol{x})=N_{n} U_{n}(\boldsymbol{x})$, $N_{n}$ is the normalization constant. Equation (65) shows $\psi_{n}(\boldsymbol{x})$ is characterized by five quantum numbers, besides $n$ they are $p_{z}, p_{\perp}, \sigma$, and $\tau$. $\tau= \pm 1$ is defined by $E=$ $\tau \sqrt{\boldsymbol{p}^{2}+m^{2}}$. To save writing, we keep only one subscript $n$ to be the representative of these five quantum numbers. To find the normalization constant, we have to complete the integration

$$
I=\int U_{n}^{\dagger}(\boldsymbol{x}) U_{n^{\prime}}(\boldsymbol{x}) \mathrm{d}^{3} x
$$

Since $U_{n}(\boldsymbol{x})$ is an eigenfunction of an Hermite operator with eigenvalue $\varepsilon_{n}, I$ is nonzero only when $\varepsilon_{n}=\varepsilon_{n^{\prime}}$.

Eq. (65) shows that the coordinates appeared in two factors of the integrand above are different. To complete the integration we have to choose one common set of coordinates in both factors. Graf formula [15, 16] solves this problem. Take the cylindric coordinates $\rho, \varphi, z$ for $\boldsymbol{x}$ in both factors, and use the formula

$$
\begin{aligned}
& \mathrm{J}_{n}\left(p_{\perp} \rho^{\prime}\right) \mathrm{e}^{-\mathrm{i} n \varphi^{\prime}} \\
& =\sum_{\nu=-\infty}^{\infty} \mathrm{J}_{n-\nu}\left(p_{\perp} R\right) \mathrm{J}_{\nu}\left(p_{\perp} \rho\right) \mathrm{e}^{-\mathrm{i} \nu \varphi} \mathrm{e}^{-\mathrm{i}(n-\nu)\left(k_{c} z-\frac{\pi}{2}\right)},
\end{aligned}
$$

in which $R=\frac{e A_{c}}{k_{c}\left(E-p_{z}\right)}$ is of the length dimension. We also define $R^{\prime}=\frac{e A_{c}}{k_{c}\left(E^{\prime}-p_{z}^{\prime}\right)}$ for following derivations. The remaining calculation becomes elementary, though is still tedious. Main steps are shown in the appendix. The result is

$$
I=\frac{4 \pi^{2}}{p_{\perp}} \delta\left(p_{\perp}-p_{\perp}^{\prime}\right) \delta\left(p_{z}-p_{z}^{\prime}\right) \delta_{n, n^{\prime}} \delta_{\sigma, \sigma^{\prime}} \delta_{\tau, \tau^{\prime}} .
$$

It is simple and nice. The normalization constant $N_{n}=$ $\frac{1}{2 \pi}$ makes eigenfunctions $\psi_{n}(\boldsymbol{x})=\frac{1}{2 \pi} U_{n}(\boldsymbol{x})$ satisfy the orthonormal relations

$$
\int \psi_{n}^{\dagger}(\boldsymbol{x}) \psi_{n^{\prime}}(\boldsymbol{x}) \mathrm{d}^{3} x=\frac{1}{p_{\perp}} \delta\left(p_{\perp}-p_{\perp}^{\prime}\right) \delta\left(p_{z}-p_{z}^{\prime}\right) \delta_{n, n^{\prime}} \delta_{\sigma, \sigma^{\prime}} \delta_{\tau, \tau^{\prime}} .
$$

A careful analysis further shows, that the orthonormal set $\left[\psi_{n}(\boldsymbol{x})\right]$ is complete.

Expand the electron field function $\Psi(\boldsymbol{x})$ in terms of $\left[\psi_{n}(\boldsymbol{x})\right]$. Writing $\psi_{n}(\boldsymbol{x})$ in the form $\psi_{n, \sigma, \tau}\left(p_{z}, p_{\perp} ; \boldsymbol{x}\right)$, defining

$$
\left.\begin{array}{l}
U_{n, \sigma}\left(p_{z}, p_{\perp} ; \boldsymbol{x}\right) \equiv \psi_{n, \sigma, 1}\left(p_{z}, p_{\perp} ; \boldsymbol{x}\right) \\
V_{n, \sigma}\left(p_{z}, p_{\perp} ; \boldsymbol{x}\right) \equiv \psi_{-n,-\sigma,-1}\left(-p_{z}, p_{\perp} ; \boldsymbol{x}\right)
\end{array}\right\}
$$

we have

$$
\begin{aligned}
\Psi(\boldsymbol{x}) & =\int_{-\infty}^{\infty} \mathrm{d} p_{z} \int_{0}^{\infty} p_{\perp} \mathrm{d} p_{\perp} \sum_{n=-\infty}^{\infty} \sum_{\sigma= \pm 1}\left[c_{n, \sigma}\left(p_{z}, p_{\perp}\right) U_{n, \sigma}\left(p_{z}, p_{\perp} ; \boldsymbol{x}\right)\right. \\
& \left.+d_{n, \sigma}^{\dagger}\left(p_{z}, p_{\perp}\right) V_{n, \sigma}\left(p_{z}, p_{\perp} ; \boldsymbol{x}\right)\right] .
\end{aligned}
$$

Using the orthonomal relations (72) we obtain expansion coefficients

$$
\left.\begin{array}{l}
c_{n, \sigma}\left(p_{z}, p_{\perp}\right)=\int U_{n, \sigma}^{\dagger}\left(p_{z}, p_{\perp} ; \boldsymbol{x}\right) \Psi(\boldsymbol{x}) \mathrm{d}^{3} x \\
d_{n, \sigma}^{\dagger}\left(p_{z}, p_{\perp}\right)=\int V_{n, \sigma}^{\dagger}\left(p_{z}, p_{\perp} ; \boldsymbol{x}\right) \Psi(\boldsymbol{x}) \mathrm{d}^{3} x
\end{array}\right\} .
$$

The quantization rules (20]21) may be equivalently written in the form

$$
\left.\begin{array}{l}
\Psi_{\iota}(\boldsymbol{x}) \Psi_{\iota^{\prime}}\left(\boldsymbol{x}^{\prime}\right)+\Psi_{\iota^{\prime}}\left(\boldsymbol{x}^{\prime}\right) \Psi_{\iota}(\boldsymbol{x})=0 \\
\Psi_{\iota}(\boldsymbol{x}) \Psi_{\iota^{\prime}}^{\dagger}\left(\boldsymbol{x}^{\prime}\right)+\Psi_{\iota^{\prime}}^{\dagger}\left(\boldsymbol{x}^{\prime}\right) \Psi_{\iota}(\boldsymbol{x})=\delta_{\iota, \iota^{\prime}} \delta\left(\boldsymbol{x}-\boldsymbol{x}^{\prime}\right)
\end{array}\right\},
$$

$\iota$ and $\iota^{\prime}=1,2,3,4$, are component indexes, which in turn means

$$
\begin{aligned}
& c_{n, \sigma}\left(p_{z}, p_{\perp}\right) c_{n^{\prime}, \sigma^{\prime}}\left(p_{z}^{\prime}, p_{\perp}^{\prime}\right)+c_{n^{\prime}, \sigma^{\prime}}\left(p_{z}^{\prime}, p_{\perp}^{\prime}\right) c_{n, \sigma}\left(p_{z}, p_{\perp}\right) \\
= & d_{n, \sigma}\left(p_{z}, p_{\perp}\right) d_{n^{\prime}, \sigma^{\prime}}\left(p_{z}^{\prime}, p_{\perp}^{\prime}\right)+d_{n^{\prime}, \sigma^{\prime}}\left(p_{z}^{\prime}, p_{\perp}^{\prime}\right) d_{n, \sigma}\left(p_{z}, p_{\perp}\right) \\
= & c_{n, \sigma}\left(p_{z}, p_{\perp}\right) d_{n^{\prime}, \sigma^{\prime}}\left(p_{z}^{\prime}, p_{\perp}^{\prime}\right)+d_{n^{\prime}, \sigma^{\prime}}\left(p_{z}^{\prime}, p_{\perp}^{\prime}\right) c_{n, \sigma}\left(p_{z}, p_{\perp}\right) \\
= & c_{n, \sigma}\left(p_{z}, p_{\perp}\right) d_{n^{\prime}, \sigma^{\prime}}^{\dagger}\left(p_{z}^{\prime}, p_{\perp}^{\prime}\right)+d_{n^{\prime}, \sigma^{\prime}}^{\dagger}\left(p_{z}^{\prime}, p_{\perp}^{\prime}\right) c_{n, \sigma}\left(p_{z}, p_{\perp}\right) \\
= & 0, \\
& c_{n, \sigma}\left(p_{z}, p_{\perp}\right) c_{n^{\prime}, \sigma^{\prime}}^{\dagger}\left(p_{z}^{\prime}, p_{\perp}^{\prime}\right)+c_{n^{\prime}, \sigma^{\prime}}^{\dagger}\left(p_{z}^{\prime}, p_{\perp}^{\prime}\right) c_{n, \sigma}\left(p_{z}, p_{\perp}\right) \\
= & d_{n, \sigma}\left(p_{z}, p_{\perp}\right) d_{n^{\prime}, \sigma^{\prime}}^{\dagger}\left(p_{z}^{\prime}, p_{\perp}^{\prime}\right)+d_{n^{\prime}, \sigma^{\prime}}^{\dagger}\left(p_{z}^{\prime}, p_{\perp}^{\prime}\right) d_{n, \sigma}\left(p_{z}, p_{\perp}\right) \\
= & \frac{1}{p_{\perp}} \delta\left(p_{\perp}-p_{\perp}^{\prime}\right) \delta\left(p_{z}-p_{z}^{\prime}\right) \delta_{n, n^{\prime}} \delta_{\sigma, \sigma^{\prime}} .
\end{aligned}
$$

They are quantization rules for the electron field in a laser. The 'vacuum state' $\left|\left[a_{i^{\prime} c}\left(\boldsymbol{k}^{\prime}\right)\right] ; 0\right\rangle$ in this formulation is therefore defined, besides (35), by

$$
\left.\begin{array}{l}
c_{n, \sigma}\left(p_{z}, p_{\perp}\right)\left|\left[a_{i^{\prime} c}\left(\boldsymbol{k}^{\prime}\right)\right] ; 0\right\rangle=0 \\
d_{n, \sigma}\left(p_{z}, p_{\perp}\right)\left|\left[a_{i^{\prime} c}\left(\boldsymbol{k}^{\prime}\right)\right] ; 0\right\rangle=0
\end{array}\right\},
$$

showing that the numbers of electron and positron in vacuum states are zeros. Writing $\varepsilon_{n}$ in the form $\varepsilon_{n, \sigma, \tau}\left(p_{z}, p_{\perp}\right)$, taking the normal product, we see the unperturbed Hamiltonian

$$
\begin{aligned}
& H_{0 r+}= \\
& \int_{-\infty}^{\infty} \mathrm{d} p_{z} \int_{0}^{\infty} p_{\perp} \mathrm{d} p_{\perp} \sum_{n=-\infty \sigma= \pm 1}^{\infty} \sum_{n, \sigma, 1}\left(p_{z}, p_{\perp}\right) c_{n, \sigma}^{\dagger}\left(p_{z}, p_{\perp}\right) c_{n, \sigma}\left(p_{z}, p_{\perp}\right) \\
& \left.-\varepsilon_{-n,-\sigma,-1}\left(-p_{z}, p_{\perp}\right) d_{n, \sigma}^{\dagger}\left(p_{z}, p_{\perp}\right) d_{n, \sigma}\left(p_{z}, p_{\perp}\right)\right] .
\end{aligned}
$$

Consider, once again, a time dependent unitary transformation generated by the operator $\mathrm{e}^{\mathrm{i} H_{0_{r+} t}}$. It transforms the state vector |\rangle and the operator $O$ into

$$
|\rangle_{(r i)}=\mathrm{e}^{\mathrm{i} H_{0 r+} t}|\rangle \text { and } O_{(r i)}=\mathrm{e}^{\mathrm{i} H_{0 r+}{ }^{t}} O \mathrm{e}^{-\mathrm{i} H_{0 r+} t}
$$

respectively. The time dependence of the state vector now is governed by the equation

$$
\mathrm{i} \frac{\mathrm{d}|t\rangle_{(r i)}}{\mathrm{d} t}=H_{(r i)}^{\prime}|t\rangle_{(r i)},
$$

in which

$$
\begin{aligned}
& H_{(r i)}^{\prime}=e \int \mathrm{d}^{3} x \int \mathrm{d}^{3} k \sum_{i} \Psi_{(r i)}^{\dagger}\left[a_{i}^{\prime}(\boldsymbol{k}) \frac{\boldsymbol{\alpha} \cdot \boldsymbol{e}_{i r} \mathrm{e}^{\mathrm{i}\left(\boldsymbol{k}_{r} \cdot \boldsymbol{x}-k t\right)}}{\sqrt{(2 \pi)^{3} 2 k}}\right. \\
& \left.+{a_{i}^{\prime \dagger}}^{\dagger}(\boldsymbol{k}) \frac{\boldsymbol{\alpha} \cdot \boldsymbol{e}_{i r}^{*} \mathrm{e}^{-\mathrm{i}\left(\boldsymbol{k}_{r} \cdot \boldsymbol{x}-k t\right)}}{\sqrt{(2 \pi)^{3} 2 k}}\right] \Psi_{(r i)}
\end{aligned}
$$


is the interaction Hamiltonian in this picture, with

$$
\begin{aligned}
& \Psi_{(r i)}(\boldsymbol{x}, t)=\int_{-\infty}^{\infty} \mathrm{d} p_{z} \int_{0}^{\infty} p_{\perp} \mathrm{d} p_{\perp} \sum_{n=-\infty \sigma= \pm 1}^{\infty} \sum_{n, \sigma}\left[c_{n}\left(p_{z}, p_{\perp}\right)\right. \\
& \times \mathrm{e}^{-\mathrm{i} \varepsilon_{n, \sigma, 1}\left(p_{z}, p_{\perp}\right) t} U_{n, \sigma}\left(p_{z}, p_{\perp} ; \boldsymbol{x}\right)+d_{n, \sigma}^{\dagger}\left(p_{z}, p_{\perp}\right) \\
& \left.\times \mathrm{e}^{-\mathrm{i} \varepsilon_{-n,-\sigma,-1}\left(-p_{z}, p_{\perp}\right) t} V_{n, \sigma}\left(p_{z}, p_{\perp} ; \boldsymbol{x}\right)\right] .
\end{aligned}
$$

This picture is called the rotation-interaction picture, and denoted by the subscript $(r i)$.

To be complete, we have to add the Coulomb energy (3) to the interaction Hamiltonian. In the rotationinteraction picture, it is to add the right hand side of

$$
\begin{gathered}
H_{c(r i)}=\frac{1}{2} \int \mathrm{d}^{3} x \int \mathrm{d}^{3} x^{\prime} \Psi_{(r i)}^{\dagger}(\boldsymbol{x}) \Psi_{(r i)}(\boldsymbol{x}) \\
\times \frac{\alpha}{\left|\boldsymbol{x}-\boldsymbol{x}^{\prime}\right|} \Psi_{(r i)}^{\dagger}\left(\boldsymbol{x}^{\prime}\right) \Psi_{(r i)}\left(\boldsymbol{x}^{\prime}\right) .
\end{gathered}
$$

to the right hand side of (83). This is important to maintain the gauge and Lorentz invariance of the theory. Of course, in applications, we may ignore this term when the correction of the first order of $\sqrt{\alpha}$ is enough.

\section{APPLICATION TO THE LASER ELECTRON SCATTERING}

Consider the laser electron collision, in the course a photon other than those in the laser is created. This is a laser Compton scattering. Suppose a circularly polarized laser propagates along the $z$-direction, as shown in eq.(37). A beam of electrons moves along the opposite direction. After collision a photon of wave vector $\boldsymbol{k}^{\prime}$ is created, and an electron of momentum $\boldsymbol{p}$ in the beam is scattered to a state of momentum $\boldsymbol{p}^{\prime}$. Since the laser here is in a specified mode of amplitude $A_{c}$, the label $\left[a_{i^{\prime} c}\left(\boldsymbol{k}^{\prime}\right)\right]$ of the 'vacuum state' in eqs. (35) and (79) will be simplified to $A_{c}$, or further to $A$. The vacuum state will be denoted by $|A ; 0\rangle$ in the following. Consider the transition from the state $\left|n, \sigma, p_{z}, 0\right\rangle=c_{n, \sigma}^{\dagger}\left(p_{z}, 0\right)|A ; 0\rangle$ to the state $\left|\boldsymbol{k}^{\prime}, \boldsymbol{e}_{i}^{\prime} ; n^{\prime}, \sigma^{\prime}, p_{z}^{\prime}, p_{\perp}^{\prime}\right\rangle=a_{i}^{\prime \dagger}\left(\boldsymbol{k}^{\prime}\right) c_{n^{\prime} \sigma^{\prime}}^{\dagger}\left(p_{z}^{\prime}, p_{\perp}^{\prime}\right)|A ; 0\rangle$. The interaction matrix element is

$$
\begin{aligned}
& \left\langle p_{\perp}^{\prime}, p_{z}^{\prime}, \sigma^{\prime}, n^{\prime} ; \boldsymbol{e}_{i}^{\prime}, \boldsymbol{k}^{\prime}\left|H_{(r i)}^{\prime}\right| n, \sigma, p_{z}, 0\right\rangle \\
& =\mathrm{e}^{\mathrm{i}\left[\varepsilon_{n^{\prime}, \sigma^{\prime}, 1}\left(p_{z}^{\prime}, p_{\perp}^{\prime}\right)+k^{\prime}-\varepsilon_{n, \sigma, 1}\left(p_{z}, 0\right)\right] t} \int U_{n^{\prime}, \sigma^{\prime}}^{\dagger}\left(p_{z}^{\prime}, p_{\perp}^{\prime} ; \boldsymbol{x}\right) \\
& \times \frac{e \boldsymbol{\alpha} \cdot \boldsymbol{e}_{i r}^{\prime *} \mathrm{e}^{-\mathrm{i} \boldsymbol{k}_{r}^{\prime} \cdot \boldsymbol{x}}}{\sqrt{(2 \pi)^{3} 2 k^{\prime}}} U_{n, \sigma}\left(p_{z}, 0 ; \boldsymbol{x}\right) \mathrm{d}^{3} x
\end{aligned}
$$

From eq. (59) we see

$$
\begin{aligned}
& U_{n, \sigma}\left(p_{z}, 0 ; \boldsymbol{x}\right)=\delta_{n, 0} U_{0, \sigma}\left(p_{z}, 0 ; \boldsymbol{x}\right) \\
& =\frac{1}{2 \pi} \mathrm{e}^{\mathrm{i}\left(p_{z}+\frac{e A}{2} R k\right) z}\left\{1+k R\left[\alpha_{x} \cos k z+\alpha_{y} \sin k z\right.\right. \\
& \left.\left.+\mathrm{i}\left(\Sigma_{y} \cos k z-\Sigma_{x} \sin k z\right)\right] / 2\right\} u_{\sigma}(0),
\end{aligned}
$$

the subscript $c$ of $k_{c}$ is also omitted. What we have to calculate is

$$
\begin{aligned}
& \Theta_{i}=\int_{0}^{\infty} \rho \mathrm{d} \rho \int_{0}^{2 \pi} \mathrm{d} \varphi u_{\sigma^{\prime}}^{\dagger}(0)\left\{\mathrm{i}^{-n^{\prime}} \mathrm{J}_{n^{\prime}}\left(p_{\perp}^{\prime} \rho^{\prime}\right) \mathrm{e}^{\mathrm{i} n^{\prime} \varphi^{\prime}} \mathrm{P}_{+}^{\prime}\right. \\
& \left.+\mathrm{i}^{-\left(n^{\prime}-\sigma^{\prime}\right)} \mathrm{J}_{n^{\prime}-\sigma^{\prime}}\left(p_{\perp}^{\prime} \rho^{\prime}\right) \mathrm{e}^{\mathrm{i}\left(n^{\prime}-\sigma^{\prime}\right) \varphi^{\prime}} \mathrm{P}_{-}^{\prime}\right\} \Xi_{i} u_{\sigma}(0) \\
& \times \sum_{n "=-\infty}^{\infty} \mathrm{i}^{-n "} \mathrm{e}^{\mathrm{i} n " \varphi_{k_{r}^{\prime}}} \mathrm{J}_{n} "\left(k_{\perp}^{\prime} \rho\right) \mathrm{e}^{-\mathrm{i} n^{\prime \prime} \varphi}
\end{aligned}
$$

with $\mathrm{P}_{ \pm}^{\prime} \equiv \frac{1 \pm \sigma^{\prime} \Sigma_{z}}{2}$, and

$$
\begin{aligned}
\Xi_{i} & \equiv\left\{1+\frac{k R^{\prime}}{2}\left[\alpha_{x} \cos k z+\alpha_{y} \sin k z-\mathrm{i}\left(\Sigma_{y} \cos k z-\Sigma_{x} \sin k z\right)\right]\right\} \alpha_{i} \\
& \times\left\{1+\frac{k R}{2}\left[\alpha_{x} \cos k z+\alpha_{y} \sin k z+\mathrm{i}\left(\Sigma_{y} \cos k z-\Sigma_{x} \sin k z\right)\right]\right\},
\end{aligned}
$$

$i=x, y, z \cdot \varphi_{k_{r}^{\prime}}=\varphi_{k^{\prime}}+k t$ is the longitude of $\boldsymbol{k}_{r}^{\prime}$, it is the angle between the projection of $\boldsymbol{k}_{r}^{\prime}$ on the $x-y$ plane and the $x$-axis. $\varphi_{k^{\prime}}$ is the longitude of $\boldsymbol{k}^{\prime}$, which is time independent. The calculation is elementary but tedious. The result is

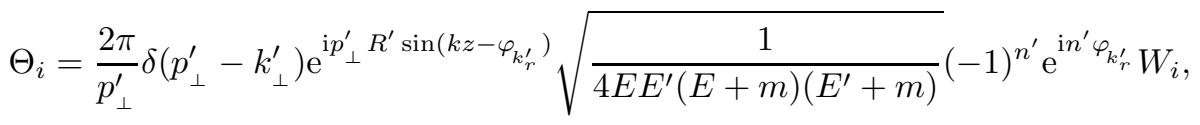




$$
\begin{aligned}
& W_{x}= {\left[\frac{k}{2}\left(p_{z}-E-m\right)\left(p_{z}^{\prime}-E^{\prime}-m\right)\left(R \mathrm{e}^{\mathrm{i} \sigma k z}+R^{\prime} \mathrm{e}^{-\mathrm{i} \sigma k z}\right)-\mathrm{e}^{-\mathrm{i} \sigma \varphi_{k_{r}^{\prime}}} p_{\perp}^{\prime}(E+m)\right] \delta_{\sigma, \sigma^{\prime}} } \\
&+\left.+\sigma p_{z}\left(E^{\prime}+m\right)-p_{z}^{\prime}(E+m)-\mathrm{e}^{\mathrm{i} \sigma \varphi_{k_{r}^{\prime}}} \frac{k}{2}\left(p_{z}-E-m\right) p_{\perp}^{\prime}\left(R \mathrm{e}^{\mathrm{i} \sigma k z}+R^{\prime} \mathrm{e}^{-\mathrm{i} \sigma k z}\right)\right] \delta_{\sigma,-\sigma^{\prime}}, \\
& W_{y}= \mathrm{i}\left\{\sigma\left[\frac{k}{2}\left(p_{z}-E-m\right)\left(p_{z}^{\prime}-E^{\prime}-m\right)\left(R^{\prime} \mathrm{e}^{-\mathrm{i} \sigma k z}-R \mathrm{e}^{\mathrm{i} \sigma k z}\right)-\mathrm{e}^{-\mathrm{i} \sigma \varphi_{k_{r}^{\prime}}} p_{\perp}^{\prime}(E+m)\right] \delta_{\sigma, \sigma^{\prime}}\right. \\
&\left.+\left[p_{z}\left(E^{\prime}+m\right)-p_{z}^{\prime}(E+m)-\mathrm{e}^{\mathrm{i} \sigma \varphi_{k_{r}^{\prime}}} \frac{k}{2}\left(p_{z}-E-m\right) p_{\perp}^{\prime}\left(R^{\prime} \mathrm{e}^{-\mathrm{i} \sigma k z}-R \mathrm{e}^{\mathrm{i} \sigma k z}\right)\right] \delta_{\sigma,-\sigma^{\prime}}\right\}, \\
& W_{z}=\left\{\left(E^{\prime}+m\right) p_{z}+(E+m) p_{z}^{\prime}+\frac{k^{2} R R^{\prime}}{2}\left(p_{z}-E-m\right)\left(p_{z}^{\prime}-E^{\prime}-m\right)\right. \\
&\left.-\frac{k p_{\perp}^{\prime}}{2} \mathrm{e}^{\mathrm{i} \sigma\left(k z-\varphi_{k_{r}^{\prime}}\right)}\left[\left(R+R^{\prime}\right)(E+m)-\left(R-R^{\prime}\right) p_{z}\right]\right\} \delta_{\sigma, \sigma^{\prime}}-\left\{\left[\frac{k^{2} R R^{\prime}}{2}\left(p_{z}-E-m\right)+E+m\right] p_{\perp} \mathrm{e}^{\mathrm{i} \sigma \varphi_{k_{r}^{\prime}}}\right. \\
&\left.-\frac{k \mathrm{e}^{\mathrm{i} \sigma k z}}{2}\left[R\left(p_{z}-E-m\right)\left(p_{z}^{\prime}+E^{\prime}+m\right)-R^{\prime}\left(p_{z}+E+m\right)\left(p_{z}^{\prime}-E^{\prime}-m\right)\right]\right\} \sigma \delta_{\sigma,-\sigma^{\prime}} .
\end{aligned}
$$

Take $\boldsymbol{e}_{1}^{\prime}=\cos \theta\left(\cos \varphi_{k^{\prime}} \boldsymbol{x}_{0}+\sin \varphi_{k^{\prime}} \boldsymbol{y}_{0}\right)-\sin \theta \boldsymbol{z}_{0}$ and $\boldsymbol{e}_{2}^{\prime}=-\sin \varphi_{k^{\prime}} \boldsymbol{x}_{0}+\cos \varphi_{k^{\prime}} \boldsymbol{y}_{0}$ to be a pair of orthonormal polarization vectors orthogonal to the wave vector $\boldsymbol{k}^{\prime}$, in which $\theta$ is the angle between $\boldsymbol{k}^{\prime}$ and the $z$ axis. We have

$$
\begin{aligned}
& \boldsymbol{\Theta} \cdot \boldsymbol{e}_{i r}^{\prime *}=\frac{2 \pi}{p_{\perp}^{\prime}} \delta\left(p_{\perp}^{\prime}-k_{\perp}^{\prime}\right) \mathrm{e}^{\mathrm{i} p_{\perp}^{\prime} R^{\prime} \sin \left(k z-\varphi_{k_{r}^{\prime}}\right)} \\
& \times \sqrt{\frac{1}{4 E E^{\prime}(E+m)\left(E^{\prime}+m\right)}(-1)^{n^{\prime}} \mathrm{e}^{\mathrm{i} n^{\prime} \varphi_{k_{r}^{\prime}}}} \\
& \times\left\{\delta_{\sigma, \sigma^{\prime}} \sum_{\nu=0, \pm 1} F_{i}^{(\nu)} \mathrm{e}^{\mathrm{i} \nu\left[k(z-t)-\varphi_{k^{\prime}}\right]}\right. \\
& \left.+\delta_{\sigma,-\sigma^{\prime}} \sum_{\nu=0, \pm 1} G_{i}^{(\nu)} \mathrm{e}^{\mathrm{i} \nu\left[k(z-t)-\varphi_{k^{\prime}}\right]} \mathrm{e} \mathrm{e}^{\mathrm{i} \sigma \varphi_{k_{r}^{\prime}}}\right\},
\end{aligned}
$$

$i=1,2$, in which

$$
\left.\begin{array}{l}
F_{1}^{(0)}=-\cos \theta p_{\perp}^{\prime}(E+m)-\sin \theta\left[\left(E^{\prime}+m\right) p_{z}\right. \\
\left.+(E+m) p_{z}^{\prime}+\frac{1}{2} k^{2} R R^{\prime}\left(p_{z}-E-m\right)\left(p_{z}^{\prime}-E^{\prime}-m\right)\right], \\
F_{1}^{(\sigma)}=\frac{k}{2}\left\{\cos \theta R\left(p_{z}-E-m\right)\left(p_{z}^{\prime}-E^{\prime}-m\right)\right. \\
+\sin \theta p_{\perp}^{\prime}\left[\left(R+R^{\prime}\right)(E+m)-\left(R-R^{\prime}\right) p_{z}\right\}, \\
F_{1}^{(-\sigma)}=\frac{k}{2} \cos \theta R^{\prime}\left(p_{z}-E-m\right)\left(p_{z}^{\prime}-E^{\prime}-m\right), \\
G_{1}^{(0)}=\sigma\left\{\cos \theta\left[p_{z}\left(E^{\prime}+m\right)-p_{z}^{\prime}(E+m)\right]\right. \\
\left.+\sin \theta p_{\perp}^{\prime}\left[\frac{k^{2} R R^{\prime}}{2}\left(p_{z}-E-m\right)+E+m\right]\right\}, \\
G_{1}^{(\sigma)}=-\frac{\sigma k}{2}\left\{\cos \theta R p_{\perp}^{\prime}\left(p_{z}-E-m\right)\right. \\
+\sin \theta\left[R\left(p_{z}-E-m\right)\left(p_{z}^{\prime}+E^{\prime}+m\right)\right. \\
\left.\left.-R^{\prime}\left(p_{z}+E+m\right)\left(p_{z}^{\prime}-E^{\prime}-m\right)\right]\right\}, \\
G_{1}^{(-\sigma)}=-\frac{\sigma k}{2} \cos \theta R^{\prime} p_{\perp}^{\prime}\left(p_{z}-E-m\right),
\end{array}\right\}
$$

$$
\begin{aligned}
& F_{2}^{(0)}=-\mathrm{i} \sigma p_{\perp}^{\prime}(E+m), \\
& F_{2}^{(\sigma)}=-\mathrm{i} \sigma \frac{k R}{2}\left(p_{z}-E-m\right)\left(p_{z}^{\prime}-E^{\prime}-m\right), \\
& F_{2}^{(-\sigma)}=\mathrm{i} \sigma \frac{k R^{\prime}}{2}\left(p_{z}-E-m\right)\left(p_{z}^{\prime}-E^{\prime}-m\right), \\
& G_{2}^{(0)}=\mathrm{i}\left[p_{z}\left(E^{\prime}+m\right)-p_{z}^{\prime}(E+m)\right] \\
& G_{2}^{(\sigma)}=\mathrm{i} \frac{k R}{2} p_{\perp}^{\prime}\left(p_{z}-E-m\right), \\
& G_{2}^{(-\sigma)}=-\mathrm{i} \frac{k R^{\prime}}{2} p_{\perp}^{\prime}\left(p_{z}-E-m\right) .
\end{aligned}
$$

Substituting (94) into (86), using (62) once again, we obtain

$$
\begin{aligned}
& \left\langle p_{\perp}^{\prime}, p_{z}^{\prime}, \sigma^{\prime}, n^{\prime} ; \boldsymbol{e}_{i}^{\prime}, \boldsymbol{k}^{\prime}\left|H_{(r i)}^{\prime}\right| n, \sigma, p_{z}, 0\right\rangle=\frac{e \delta\left(p_{\perp}^{\prime}-k_{\perp}^{\prime}\right)}{\sqrt{(2 \pi)^{3} 2 k^{\prime}} p_{\perp}^{\prime}} \\
& \times \sqrt{\frac{1}{4 E E^{\prime}(E+m)\left(E^{\prime}+m\right)}} \sum_{\mathcal{N}=-\infty}^{\infty} \sum_{\nu=0, \pm 1} \mathrm{~J}_{\mathcal{N}-\nu}\left(p_{\perp}^{\prime} R^{\prime}\right) \\
& \times\left[\delta_{\sigma, \sigma^{\prime}} F_{i}^{(\nu)} \mathrm{e}^{-\mathrm{i} \mathcal{N} \varphi_{k^{\prime}}}+\delta_{\sigma,-\sigma^{\prime}} G_{i}^{(\nu)} \mathrm{e}^{\mathrm{i}(\sigma-\mathcal{N}) \varphi_{k^{\prime}}}\right] \\
& \times \delta\left[p_{z}^{\prime}-p_{z}+\frac{e A}{2}\left(R^{\prime}-R\right) k+k_{z}^{\prime}-\mathcal{N} k\right] \\
& \times \mathrm{e}^{\mathrm{i} n^{\prime}\left(\varphi_{k^{\prime}}+\pi\right)} \mathrm{e} \mathrm{i}\left[E^{\prime}-E+\frac{e A}{2}\left(R^{\prime}-R\right) k+k^{\prime}-\mathcal{N} k\right] t .
\end{aligned}
$$

Notice, terms containing the factor $\frac{\sigma}{2}-n$ in $\varepsilon_{n}$ and the factor $\frac{\sigma^{\prime}}{2}-n^{\prime}$ in $\varepsilon_{n^{\prime}}$ disappear due to cancelations in the calculation. The matrix element is decomposed into a series of terms with different time dependence, characterized by an integer parameter $\mathcal{N}$. The resonance condition

$$
E^{\prime}-E+\frac{e A}{2}\left(R^{\prime}-R\right) k+k^{\prime}-\mathcal{N} k=0
$$

is put on variables $E^{\prime}, E, p_{z}^{\prime}, k^{\prime}, k$ and on the parameter $\mathcal{N}$, but not on $\sigma^{\prime}, \sigma, n^{\prime}$ and $n$. Therefore, transitions with same $E^{\prime}, E, p_{z}^{\prime}, k^{\prime}, k$ and $\mathcal{N}$, but different $\sigma^{\prime}, \sigma, n^{\prime}$ and $n$, may have nonzero probabilities simultaneously and be coherent with each other. This point is important in the following. In the limit $A \rightarrow 0$, this equation may be 
interpreted as energy conservation in the collision, $\mathcal{N}$ is interpreted to be the number of photons in laser absorbed during the collision. This interpretation is questionable since its ignorance of coherence and interactions between particle states. It becomes obvious when $A$ is finite.

In quantum field theory, people assumes that the interaction switches on and off infinitely slow. This is the adiabatic assumption. We take this assumption for the case with a laser interaction, so that the Gell-Mann Low theorem [17] is applicable to the equation (66). Eqs. (73) and (65) show that at the limit $A \rightarrow 0$,

$$
\begin{aligned}
& U_{n, \sigma}\left(p_{z}, p_{\perp} ; \boldsymbol{x}\right) \rightarrow U_{n, \sigma}^{(0)}\left(p_{z}, p_{\perp} ; \boldsymbol{x}\right) \equiv \frac{\mathrm{e}^{\mathrm{i} p_{z} z}}{2 \pi} \\
& \times\left[\mathrm{i}^{n} \mathrm{~J}_{n}\left(p_{\perp} \rho\right) \mathrm{e}^{-\mathrm{i} n \varphi} \mathrm{P}_{+}+\mathrm{i}^{n-\sigma} \mathrm{J}_{n-\sigma}\left(p_{\perp} \rho\right) \mathrm{e}^{-\mathrm{i}(n-\sigma) \varphi} \mathrm{P}\right] u_{\sigma}(0) .
\end{aligned}
$$

This is a solution of Dirac equation for a free electron, and

$$
\sum_{n=-\infty}^{\infty} \frac{\mathrm{e}^{\mathrm{i} n \varphi_{p}}}{\sqrt{2 \pi}} U_{n, \sigma}^{(0)}\left(p_{z}, p_{\perp} ; \boldsymbol{x}\right)=\frac{1}{\sqrt{(2 \pi)^{3}}} \mathrm{e}^{\mathrm{i} \boldsymbol{p} \cdot \boldsymbol{x}} u
$$

is a plane wave solution of Dirac equation for a free electron of momentum $\boldsymbol{p}=p_{\perp}\left(\cos \varphi_{p} \boldsymbol{x}_{0}+\sin \varphi_{p} \boldsymbol{y}_{0}\right)+p_{z} \boldsymbol{z}_{0}$, $u$ is defined by eq.(58). In the case of $p_{\perp}=0$, we have

$$
U_{n, \sigma}^{(0)}\left(p_{z}, 0 ; \boldsymbol{x}\right)=\frac{\delta_{n, 0}}{2 \pi} \mathrm{e}^{\mathrm{i} p_{z} z} u .
$$

It is nonzero only when $n=0$. In this case, it is already a plane wave of electron with momentum $\boldsymbol{p}=p_{z} \boldsymbol{z}_{0}$.

Suppose a free electron of momentum $\boldsymbol{p}=p_{z} \boldsymbol{z}_{0}$ and spin $\sigma$ comes from remote past and meets a laser on the way. It evolves according to Gell-Mann Low theorem into the state $U_{n, \sigma}\left(p_{z}, 0 ; \boldsymbol{x}\right)$. The above analysis shows, this state may transit to a superposition $\sum_{n^{\prime}=-\infty}^{\infty} \frac{\mathrm{e}^{\mathrm{i} n^{\prime} \varphi_{p}}}{\sqrt{2 \pi}} U_{n^{\prime}, \sigma^{\prime}}\left(p_{z}^{\prime}, p_{\perp}^{\prime} ; \boldsymbol{x}\right)$ of electron states in the laser due to the electromagnetic interaction $H_{(r i)}^{\prime}$, and emit a photon of momentum $\boldsymbol{k}^{\prime}$. This superposition of electron states evolves once again in the laser, into the state $\frac{1}{\sqrt{(2 \pi)^{3}}} \mathrm{e}^{\mathrm{i} \boldsymbol{p}^{\prime} \cdot \boldsymbol{x}} u_{\sigma^{\prime}}$ of a free electron when goes to the remote future. In this process, the initial state is $\left|N, \boldsymbol{k}^{\prime}, \boldsymbol{e}_{i}^{\prime} ; \boldsymbol{p}, \sigma\right\rangle=\frac{1}{\sqrt{N !}}{ }_{i}^{\prime \dagger N}\left(\boldsymbol{k}^{\prime}\right) c_{0, \sigma}^{\dagger}\left(p_{z}, 0\right)|A ; 0\rangle$, with $\boldsymbol{p}=p_{z} \boldsymbol{z}_{0} . \quad N$ is the number of photons of momentum $\boldsymbol{k}^{\prime} \neq \boldsymbol{k}$ before the emission. The final state is

$$
\begin{aligned}
& \left|N+1, \boldsymbol{k}^{\prime}, \boldsymbol{e}_{i}^{\prime} ; \boldsymbol{p}^{\prime}, \sigma^{\prime}\right\rangle=\frac{1}{\sqrt{(N+1) !}} \\
& \times{a_{i}^{\prime}}^{\dagger N+1}\left(\boldsymbol{k}^{\prime}\right) \sum_{n^{\prime}=-\infty}^{\infty} \frac{\mathrm{e}^{\mathrm{i} n^{\prime} \varphi_{p^{\prime}}}}{\sqrt{2 \pi}} c_{n^{\prime}, \sigma^{\prime}}^{\dagger}\left(p_{z}^{\prime}, p_{\perp}^{\prime}\right)|A ; 0\rangle .
\end{aligned}
$$

The interaction matrix element is

$$
\begin{aligned}
& \left\langle\sigma^{\prime}, \boldsymbol{p}^{\prime} ; \boldsymbol{e}_{i}^{\prime}, \boldsymbol{k}^{\prime}, N+1\left|H_{(r i)}^{\prime}\right| N, \boldsymbol{k}^{\prime}, \boldsymbol{e}_{i}^{\prime} ; \boldsymbol{p}, \sigma\right\rangle=\frac{e}{2 \pi \sqrt{2 k^{\prime}}} \\
& \times \sqrt{\frac{N+1}{4 E E^{\prime}(E+m)\left(E^{\prime}+m\right)}} \sum_{\mathcal{N}=-\infty}^{\infty} \sum_{\nu=0, \pm 1} \mathrm{~J}_{\mathcal{N}-\nu}\left(p_{\perp}^{\prime} R^{\prime}\right) \\
& \times\left[\delta_{\sigma, \sigma^{\prime}} F_{i}^{(\nu)} \mathrm{e}^{-\mathrm{i} \mathcal{N} \varphi_{k^{\prime}}}+\delta_{\sigma,-\sigma^{\prime}} G_{i}^{(\nu)} \mathrm{e}^{\mathrm{i}(\sigma-\mathcal{N}) \varphi_{k^{\prime}}}\right] \\
& \times \delta\left[\boldsymbol{p}^{\prime}+\boldsymbol{k}^{\prime}-\boldsymbol{p}-\mathcal{N} \boldsymbol{k}+\frac{e A}{2}\left(R^{\prime}-R\right) \boldsymbol{k}\right] \\
& \times \mathrm{e}^{\mathrm{i}\left[E^{\prime}-E+\frac{e A}{2}\left(R^{\prime}-R\right) k+k^{\prime}-\mathcal{N} k\right] t},
\end{aligned}
$$

in which

$$
\begin{aligned}
& \delta\left[\boldsymbol{p}^{\prime}+\boldsymbol{k}^{\prime}-\boldsymbol{p}-\mathcal{N} \boldsymbol{k}+\frac{e A}{2}\left(R^{\prime}-R\right) \boldsymbol{k}\right] \\
& =\frac{\delta\left(p_{\perp}^{\prime}-k_{\perp}^{\prime}\right)}{p_{\perp}^{\prime}} \delta\left(\varphi_{k^{\prime}}+\pi-\varphi_{p^{\prime}}\right) \\
& \times \delta\left[p_{z}^{\prime}-p_{z}+\frac{e A}{2}\left(R^{\prime}-R\right) k+k_{z}^{\prime}-\mathcal{N} k\right]
\end{aligned}
$$

is a 3 -dimensional $\delta$-function.

In the first order perturbation, the transition amplitude of the process is

$$
\begin{aligned}
& \left\langle\sigma^{\prime}, \boldsymbol{p}^{\prime} ; \boldsymbol{e}_{i}^{\prime}, \boldsymbol{k}^{\prime}, N+1|T| N, \boldsymbol{k}^{\prime}, \boldsymbol{e}_{i}^{\prime} ; \boldsymbol{p}, \sigma\right\rangle=-\mathrm{i} \int_{-\infty}^{\infty}\left\langle\sigma^{\prime}, \boldsymbol{p}^{\prime} ; \boldsymbol{e}_{i}^{\prime}, \boldsymbol{k}^{\prime}, N+1\left|H_{(r i)}^{\prime}\right| N, \boldsymbol{k}^{\prime}, \boldsymbol{e}_{i}^{\prime} ; \boldsymbol{p}, \sigma\right\rangle \mathrm{d} t=-\mathrm{i} \frac{e}{\sqrt{2 k^{\prime}}} \\
& \left.\times \sqrt{\frac{N+1}{4 E E^{\prime}(E+m)\left(E^{\prime}+m\right)}} \sum_{\mathcal{N}=-\infty \nu=0, \pm 1}^{\infty} \sum_{\mathcal{N}-\nu} \mathrm{J}_{\perp}^{\prime} R^{\prime}\right)\left[\delta_{\sigma, \sigma^{\prime}} F_{i}^{(\nu)} \mathrm{e}^{-\mathrm{i} \mathcal{N} \varphi_{k^{\prime}}}+\delta_{\sigma,-\sigma^{\prime}} G_{i}^{(\nu)} \mathrm{e}^{\mathrm{i}(\sigma-\mathcal{N}) \varphi_{k^{\prime}}}\right] \\
& \times \delta\left[\boldsymbol{p}^{\prime}+\boldsymbol{k}^{\prime}-\boldsymbol{p}-\mathcal{N} \boldsymbol{k}+\frac{e A}{2}\left(R^{\prime}-R\right) \boldsymbol{k}\right] \delta\left[E^{\prime}+k^{\prime}-E-\mathcal{N} k+\frac{e A}{2}\left(R^{\prime}-R\right) k\right] .
\end{aligned}
$$

$\delta$-functions give selection rules for non-zero transition probability. Besides eq. (98), we have

$$
\boldsymbol{p}^{\prime}+\boldsymbol{k}^{\prime}-\boldsymbol{p}-\mathcal{N} \boldsymbol{k}+\frac{e A}{2}\left(R^{\prime}-R\right) \boldsymbol{k}=0 .
$$

In the limit $A=0$, it means the usual momentum conservation of the process. The $A$-dependent term shows the coherence effect of the laser. Using these two selection 
rules we obtain

$$
k^{\prime}=\frac{\mathcal{N} k\left(E-p_{z}\right)}{E+\mathcal{N} k+\frac{e A}{2} R k-\left(p_{z}+\mathcal{N} k+\frac{e A}{2} R k\right) \cos \theta} .
$$

For given incident electrons and laser, this formula gives the direction dependence of the energy of emitted photon. For $\mathcal{N}=1$ and in the limit $A=0$, it reduces to the Compton formula for the usual Compton scattering 18, 19.

According to the collision theory, the transition probability is 13, 14]

$$
P=\left|\left\langle\sigma^{\prime}, \boldsymbol{p}^{\prime} ; \boldsymbol{e}_{i}^{\prime}, \boldsymbol{k}^{\prime}, N+1|T| N, \boldsymbol{k}^{\prime}, \boldsymbol{e}_{i}^{\prime} ; \boldsymbol{p}, \sigma\right\rangle\right|^{2} \mathrm{~d}^{3} p^{\prime} \mathrm{d}^{3} k^{\prime} .
$$

The easiest way to understand the multiplication of $\mathrm{d}^{3} p^{\prime} \mathrm{d}^{3} k^{\prime}$ is by the method of box normalization. The squares of $\delta$-functions are handled by

$$
\begin{aligned}
& \delta[\Phi(\zeta)] \mathrm{d} \zeta=\delta[\Phi(\zeta)] \mathrm{d} \Phi \frac{\mathrm{d} \zeta}{\mathrm{d} \Phi}=\frac{\mathrm{d} \zeta}{\mathrm{d} \Phi}, \\
& \text { under the condition of } \Phi(\zeta)=0
\end{aligned}
$$

and

$$
\delta(0)=\frac{1}{2 \pi} \int_{-\infty}^{\infty} \mathrm{d} \xi=\frac{\Delta \xi}{2 \pi}, \quad \text { for macroscopic } \Delta \xi .(110)
$$

The transition probability per-unit time in unit volume and unit solid angle of $\boldsymbol{k}^{\prime}$ is

$$
\begin{aligned}
& \frac{\partial^{5} P}{\partial^{3} x \partial t \partial \Omega_{k^{\prime}}}=\frac{\alpha k^{\prime}}{(2 \pi)^{3}} \frac{N+1}{4 E E^{\prime}(E+m)\left(E^{\prime}+m\right)} \\
& \times \frac{E^{\prime} k^{\prime}}{\mathcal{N} k\left(E-p_{z}\right)} \sum_{\mathcal{N}=-\infty}^{\infty} \mid \sum_{\nu=0, \pm 1} \mathrm{~J}_{\mathcal{N}-\nu}\left(p_{\perp}^{\prime} R^{\prime}\right)\left[\delta_{\sigma, \sigma^{\prime}} F_{i}^{(\nu)} \mathrm{e}^{-\mathrm{i} \mathcal{N} \varphi_{k^{\prime}}}\right. \\
& +\left.\delta_{\sigma,-\sigma^{\prime}} G_{i}^{(\nu)} \mathrm{e}^{\mathrm{i}(\sigma-\mathcal{N}) \varphi_{k^{\prime}}}\right|^{2} .
\end{aligned}
$$

In the problem of collision between particles, the cross section of the target particle is measured and calculated. It is the probability of occurrence of a given kind collision when there is an incident particle comes across a unit area. Our problem is the collision between an electron and a laser. The corresponding quantity to be measured and calculated is the probability of creating a photon in a volume of the laser by an electron coming across a unit area. We call it the cross section of that volume of the laser. In the remote past, the incident electron was in the state (101). There is a piece of probability of amount $\left|p_{z}\right| /\left[E(2 \pi)^{2}\right]$ passing across a unit area in the $x-$ $y$ plane per unit time. In our unit system, the compton wavelength of an electron is $m^{-1}$. In the unit of $\mathrm{m}^{-2}$, the differential cross section of a piece of laser of volume $m^{-3}$, in which a photon of momentum $\boldsymbol{k}^{\prime}$ is emitted in a unit solid angle by an incident electron, is

$$
\begin{aligned}
& \frac{\mathrm{d} \Sigma}{\mathrm{d} \Omega_{k^{\prime}}}=\frac{\alpha k^{\prime 2}(N+1)}{8 \pi m \mathcal{N} k\left|p_{z}\right|\left(E-p_{z}\right)(E+m)\left(E^{\prime}+m\right)} \\
& \times \sum_{\mathcal{N}=-\infty}^{\infty}\left|\sum_{\nu=0, \pm 1} \mathrm{~J}_{\mathcal{N}-\nu}\left(p_{\perp}^{\prime} R^{\prime}\right)\left[\delta_{\sigma, \sigma^{\prime}} F_{i}^{(\nu)}+\delta_{\sigma,-\sigma^{\prime}} G_{i}^{(\nu)} \mathrm{e}^{\mathrm{i} \sigma \varphi_{k^{\prime}}}\right]\right|^{2} .
\end{aligned}
$$

The cross section is decomposed into an incoherent sum of partial cross sections, each associates an integer $\mathcal{N}$, which may be imagined to be the number of photons in the laser participating the collision. A partial cross section is proportional to an absolute square of a superposition of three partial waves each contains a Bessel function factor. It forms a characteristic diffraction pattern depending on the integer $\mathcal{N}$, to be verified by experiments. This result seems to be an interpretation of the multi-photon process seen in experiments [1, 2]. However, the physics shown in our derivation is not a multi-photon process, but is an effect originated by the electron wave distortion in a laser. It is exactly handled for the case of circularly polarized laser. In the collision, only one photon is emitted directly by an electron. It is handled by perturbation. This is justified by the smallness of the fine structure constant $\alpha$. The precision of the derivation here is therefore as high as those in the usual quantum electrodynamics in vacuum.

To compare this result with the Klein-Nishina formula 20, 21] for the usual Compton scattering is interesting. Take $N=0$ and $\mathcal{N}=1$, calculate the average cross section

$$
\overline{\frac{\mathrm{d} \Sigma}{\mathrm{d} \Omega_{k^{\prime}}}}(i)=\frac{1}{2} \sum_{\sigma= \pm 1} \sum_{\sigma^{\prime}= \pm 1} \frac{\mathrm{d} \Sigma}{\mathrm{d} \Omega_{k^{\prime}}}
$$

from the formula (112). The result is still dependent on the polarization $i$ of the outgoing photon state. On the other hand, transform the Klein-Nishina formula into a coordinate frame, in which an electron moves and makes a head-on collision with a target photon. It may be easily done by Lorentz and gauge transformations [13, 22]. In a laser of amplitude $A$ and frequency $k$, the number of photons in a volume $m^{-3}$ is $\frac{k}{m}\left(\frac{e A}{m}\right)^{2} /(4 \pi \alpha)$. Multiplying the transformed Klein-Nishina formula by this number, we obtain the total average cross section

$$
\begin{aligned}
& \left.\overline{\frac{\mathrm{d} \Sigma}{\mathrm{d} \Omega_{k^{\prime}}}}(i)\right|_{K N}=\frac{\alpha\left(e A k^{\prime}\right)^{2}}{16 \pi m k\left|p_{z}\right|\left(E-p_{z}\right)}\left[\frac{\left(E-p_{z}\right) k}{\left(E-p_{z} \cos \theta\right) k^{\prime}}\right. \\
& \left.+\frac{\left(E-p_{z} \cos \theta\right) k^{\prime}}{\left(E-p_{z}\right) k}-2+4\left|\boldsymbol{e} \cdot \boldsymbol{e}_{i}^{\prime}\right|^{2}\right]
\end{aligned}
$$

of an aggregate of non-coherent photons in a volume $m^{-3}$ for a head-on incident electron. The unit of the cross section here is again $m^{-2}$. Numerical calculation shows, for given $E, k, i$ and $A$, the output from (113) with $\mathcal{N}=1$ is smaller than that from (114). The coherence of photon states in the laser depresses the transition probability. This depression in turn offers a way for measuring the coherent amplitude $A$ of a photon beam. The difference of outputs approaches zero rapidly when $A \rightarrow 0$. It is reasonable, and may be regarded as a check of our derivation here. Examples for $E / m=3 \times 10^{2}$ and $k / m=3.09 \times 10^{-6}$ are shown in fig 1 and fig, 2 relations between

$Y(i) \equiv \log _{10}\left[\left(\left.\overline{\frac{\mathrm{d} \Sigma}{\mathrm{d} \Omega_{k^{\prime}}}}(i)\right|_{K N}-\overline{\frac{\mathrm{d} \Sigma}{\mathrm{d} \Omega_{k^{\prime}}}}(i)\right) /\left.\overline{\frac{\mathrm{d} \Sigma}{\mathrm{d} \Omega_{k^{\prime}}}}(i)\right|_{K N}\right]$ and $\quad X \equiv \log _{10}(e A / m)$ are plotted. 


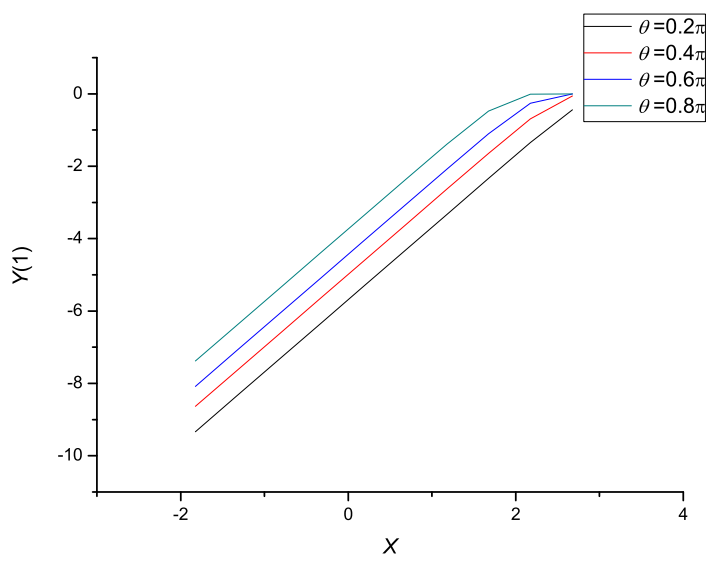

FIG. 1: Relations between $Y(1)$ and $X$, in which $E / m=$ $3 \times 10^{2}$ and $k / m=3.09 \times 10^{-6}, \theta$ is the angle between the wave vector $\boldsymbol{k}^{\prime}$ of the outgoing photon state and the wave vector $\boldsymbol{k}$ of the incident laser.

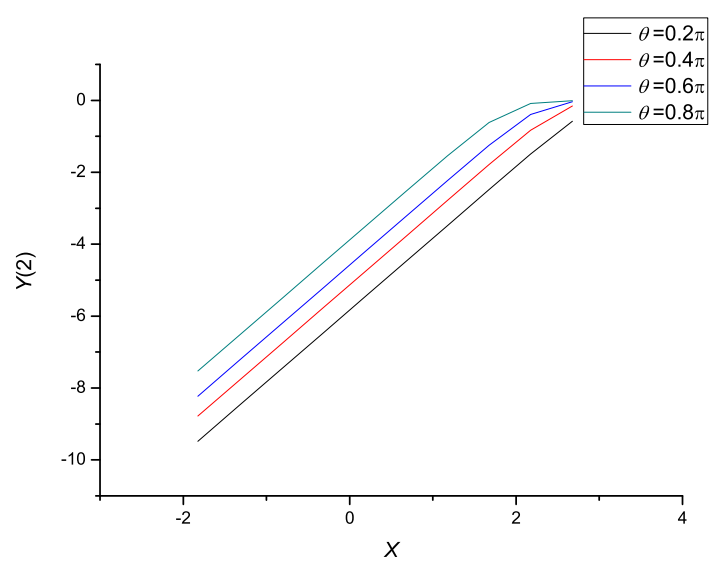

FIG. 2: Relations between $Y(2)$ and $X$, in which $E / m=$ $3 \times 10^{2}$ and $k / m=3.09 \times 10^{-6}, \theta$ is the angle between the wave vector $\boldsymbol{k}^{\prime}$ of the outgoing photon state and the wave vector $\boldsymbol{k}$ of the incident laser.

For an arbitrarily given polarization $\boldsymbol{e}^{\prime}=\sum_{i=1,2} c_{i} \boldsymbol{e}_{i}^{\prime}$ of the created photon, with $\left|c_{1}\right|^{2}+\left|c_{2}\right|^{2}=1$, the cross section (112) becomes

$$
\begin{aligned}
& \frac{\mathrm{d} \Sigma}{\mathrm{d} \Omega_{k^{\prime}}}=\frac{\alpha k^{2}(N+1)}{8 \pi m \mathcal{N} k\left|p_{z}\right|\left(E-p_{z}\right)(E+m)\left(E^{\prime}+m\right)} \\
& \times \sum_{\mathcal{N}=-\infty}^{\infty}\left|\left[\delta_{\sigma, \sigma^{\prime}} \boldsymbol{e}^{\prime *} \cdot \mathcal{F}_{\mathcal{N}}+\delta_{\sigma,-\sigma^{\prime}} \boldsymbol{e}^{\prime *} \cdot \mathcal{G}_{\mathcal{N}}\right]\right|^{2}
\end{aligned}
$$

in which

$$
\left.\begin{array}{c}
\mathcal{F}_{\mathcal{N}}=\sum_{\nu=0, \pm 1} \boldsymbol{F}^{(\nu)} \mathrm{J}_{\mathcal{N}-\nu}\left(p_{\perp}^{\prime} R^{\prime}\right) \\
\mathcal{G}_{\mathcal{N}}=\sum_{\nu=0, \pm 1} \boldsymbol{G}^{(\nu)} \mathrm{J}_{\mathcal{N}-\nu}\left(p_{\perp}^{\prime} R^{\prime}\right),
\end{array}\right\}
$$

with

$$
\boldsymbol{F}^{(\nu)} \equiv \sum_{i=1,2} F_{i}^{(\nu)} \boldsymbol{e}_{i}^{\prime}, \quad \boldsymbol{G}^{(\nu)} \equiv \sum_{i=1,2} G_{i}^{(\nu)} \mathrm{e}^{\mathrm{i} \sigma \varphi_{k^{\prime}}} \boldsymbol{e}_{i}^{\prime}
$$

For definite $\sigma, \sigma^{\prime}$ and $\mathcal{N}$, the polarization of the outgoing photon is also definite. It is $\boldsymbol{e}_{f}^{\prime} \equiv \mathcal{F}_{\mathcal{N}} / \mathcal{F}_{\mathcal{N}}$ for $\sigma^{\prime}=\sigma$ or $e_{g}^{\prime} \equiv \mathcal{G}_{\mathcal{N}} / \mathcal{G}_{\mathcal{N}}$ for $\sigma^{\prime}=-\sigma$, with $\mathcal{F}_{\mathcal{N}} \equiv \sqrt{\mathcal{F}_{\mathcal{N}}^{*} \cdot \mathcal{F}_{\mathcal{N}}}$ and $\mathcal{G}_{\mathcal{N}} \equiv \sqrt{\mathcal{G}_{\mathcal{N}}^{*} \cdot \mathcal{G}_{\mathcal{N}}}$. For an arbitrarily given polarization $\boldsymbol{e}^{\prime}$ of the outgoing photon, the average cross section (113) becomes

$$
\begin{aligned}
\overline{\frac{\mathrm{d} \Sigma}{\mathrm{d} \Omega_{k^{\prime}}}}\left(\boldsymbol{e}^{\prime}\right) & =\frac{\alpha k^{\prime 2}(N+1)}{16 \pi m \mathcal{N} k\left|p_{z}\right|\left(E-p_{z}\right)(E+m)\left(E^{\prime}+m\right)} \\
& \times \sum_{\mathcal{N}=-\infty}^{\infty} \sum_{\sigma= \pm 1} \boldsymbol{e}^{\prime *} \cdot \mathcal{Z}_{\mathcal{N}} \cdot \boldsymbol{e}^{\prime}
\end{aligned}
$$

in which

$$
\mathcal{Z}_{\mathcal{N}} \equiv \mathcal{F}_{\mathcal{N}} \mathcal{F}_{\mathcal{N}}^{*}+\mathcal{G}_{\mathcal{N}} \mathcal{G}_{\mathcal{N}}^{*}
$$

is a tensor. If the polarization of the outgoing photon is not measured, the average cross section is

$$
\begin{aligned}
& \frac{\mathrm{d} \Sigma}{\mathrm{d} \Omega_{k^{\prime}}}=\frac{\alpha k^{\prime 2}(N+1)}{16 \pi m \mathcal{N} k\left|p_{z}\right|\left(E-p_{z}\right)(E+m)\left(E^{\prime}+m\right)} \\
& \times \sum_{\mathcal{N}=-\infty}^{\infty} \sum_{\sigma= \pm 1}\left(\mathcal{F}_{\mathcal{N}}^{2}+\mathcal{G}_{\mathcal{N}}^{2}\right) .
\end{aligned}
$$

Suppose the incident electron and the outgoing photon are polarized, with certain quantum numbers $\sigma$ and $\boldsymbol{e}^{\prime}$. The state of outgoing electron is a superposition $a_{1}\left|\boldsymbol{k}^{\prime}, \boldsymbol{e}^{\prime} ; n^{\prime}, \sigma_{1}^{\prime}, p_{z}^{\prime}, p_{\perp}^{\prime}\right\rangle+a_{2}\left|\boldsymbol{k}^{\prime}, \boldsymbol{e}^{\prime} ; n^{\prime}, \sigma_{2}^{\prime}, p_{z}^{\prime}, p_{\perp}^{\prime}\right\rangle$ of states with $\sigma_{1}^{\prime}=\sigma, \sigma_{2}^{\prime}=-\sigma$, and $\left|a_{1}\right|^{2}+\left|a_{2}\right|^{2}=1$ in general. The cross section (115) becomes

$$
\begin{aligned}
& \frac{\mathrm{d} \Sigma}{\mathrm{d} \Omega_{k^{\prime}}}=\frac{\alpha k^{\prime 2}(N+1)}{8 \pi m \mathcal{N} k\left|p_{z}\right|\left(E-p_{z}\right)(E+m)\left(E^{\prime}+m\right)} \\
& \times \sum_{\mathcal{N}=-\infty}^{\infty} \boldsymbol{e}^{\prime *} \cdot \mathcal{Z}_{\mathcal{N}} \cdot \boldsymbol{e}^{\prime}\left|\chi^{\prime \dagger} \chi_{\mathcal{N}}\right|^{2},
\end{aligned}
$$

in which

$$
\chi^{\prime}=\left[\begin{array}{l}
a_{1} \\
a_{2}
\end{array}\right] \quad \text { and } \quad \chi_{\mathcal{N}}=\frac{1}{\sqrt{\boldsymbol{e}^{\prime *} \cdot \mathcal{Z}_{\mathcal{N}} \cdot \boldsymbol{e}^{\prime}}}\left[\begin{array}{l}
\boldsymbol{e}^{\prime *} \cdot \mathcal{F}_{\mathcal{N}} \\
\boldsymbol{e}^{\prime *} \cdot \mathcal{G}_{\mathcal{N}}
\end{array}\right]
$$

are normalized spinors in the $\left[\chi_{\sigma}\right]$ representation. Since the cross section is zero when $\chi^{\prime}$ is orthogonal to $\chi_{\mathcal{N}}$, the outgoing electron is polarized. Its spin state is described by the spinor $\chi_{\mathcal{N}}$.

\section{THE CASE OF LARGE $\mathcal{N}$}

The Bessel function in equation (112) may be easily worked out by its series expression when the order and the argument are small. On the other hand, 
TABLE I: Some numerical results of average cross sections calculated by use of (113) with $\theta=3.14$ and $e A / m=1.50 \times$ $10^{-2}$

\begin{tabular}{ccccc}
\hline $\mathcal{N}$ & $E / m$ & $k / m$ & $k^{\prime} / m$ & $\frac{\mathrm{d} \Sigma}{\mathrm{d} \Omega_{k^{\prime}}}(1)$ \\
\hline 1 & $7.0 \times 10^{3}$ & $3.09 \times 10^{-6}$ & 2.648 & $2.27 \times 10^{-9}$ \\
\hline 2 & $7.0 \times 10^{3}$ & $3.09 \times 10^{-6}$ & 5.28 & $9.99 \times 10^{-13}$ \\
\hline 3 & $7.0 \times 10^{3}$ & $3.09 \times 10^{-6}$ & 7.89 & $4.18 \times 10^{-16}$ \\
\hline
\end{tabular}

TABLE II: Some numerical results of average cross sections calculated by use of (113) with $\theta=3.14$ and $e A / m=10.5$

\begin{tabular}{ccccc}
\hline $\mathcal{N}$ & $E / m$ & $k / m$ & $k^{\prime} / m$ & $\frac{\mathrm{d} \Sigma}{\mathrm{d} \Omega_{k^{\prime}}}(1)$ \\
\hline 1 & $7.0 \times 10^{3}$ & $4.43 \times 10^{-9}$ & $7.76 \times 10^{-5}$ & $4.17 \times 10^{-8}$ \\
\hline 2 & $7.0 \times 10^{3}$ & $4.43 \times 10^{-9}$ & $1.55 \times 10^{-4}$ & $3.67 \times 10^{-9}$ \\
\hline 3 & $7.0 \times 10^{3}$ & $4.43 \times 10^{-9}$ & $2.33 \times 10^{-4}$ & $3.05 \times 10^{-10}$ \\
\hline 523 & $7.0 \times 10^{3}$ & $4.43 \times 10^{-9}$ & 1.90 & $1.08 \times 10^{-8}$ \\
\hline
\end{tabular}

from selection rules (98) and (106) we see $E^{\prime}-p_{z}^{\prime}=$ $E-p_{z}-k^{\prime}(1-\cos \theta)$. The argument of the Bessel function here is therefore

$$
\begin{aligned}
& p_{\perp}^{\prime} R^{\prime}=\sin \theta \frac{\mathcal{N}\left(E-p_{z}\right)}{E+\left(\mathcal{N} k+\frac{e A}{2} R k\right)(1-\cos \theta)-p_{z} \cos \theta} \\
& \times \frac{e A}{E-p_{z}-k^{\prime}(1-\cos \theta)} \\
& =\frac{\mathcal{N} e A \sin \theta}{E+\frac{e A}{2} R k(1-\cos \theta)-p_{z} \cos \theta}=\mathcal{S N}
\end{aligned}
$$

$\mathcal{S}$ is independent of $\mathcal{N}$. When $\mathcal{N}$ becomes large, the order and the argument of the Bessel function become large simultaneously. The asymptotic form for this case is well known[15, 16]. For our case, $0<\mathcal{S} \leq 1$, we have $\mathcal{S}=\operatorname{sech} \xi, \xi$ is a real number. The two term asymptotic form of the Bessel function is

$$
\begin{aligned}
& \mathrm{J}_{\mathcal{N} \pm \nu}(\mathcal{N} \operatorname{sech} \xi) \simeq \mathrm{J}_{\mathcal{N}}(\mathcal{N} \operatorname{sech} \xi) \\
& \sim \frac{\mathrm{e}^{\mathcal{N}(\tanh \xi-\xi)}}{\sqrt{2 \mathcal{N} \pi \tanh \xi}}\left[1-\left(\frac{1}{8}-\frac{5}{24} \operatorname{coth}^{2} \xi\right) \frac{1}{\mathcal{N} \tanh \xi}\right] \\
& \text { for } \nu=0, \pm 1 \text { and } \mathcal{N} \rightarrow \infty
\end{aligned}
$$

This asymptotic form makes us able to consider the case of large $\mathcal{N}$. When other parameters are fixed, $\mathcal{N}$ may be fixed by maximizing the cross section.

Some numerical results are listed in the tables, one case occupies one row. For an example, the first row of table $\llbracket$ shows the case 1 with $\mathcal{N}=1$. In this case, in the usual units, the energy of the incident electron is $7 \times 10^{3} \times$ $0.51 \mathrm{MeV}=3.57 \mathrm{GeV}$, the energy of a photon in the headon laser is $3.09 \times 10^{-6} \times 0.51 \mathrm{MeV}=1.576 \mathrm{eV}$, the energy of the emitted photon is $2.648 \times 0.51 \mathrm{MeV}=1.35 \mathrm{MeV}$, and the average differential cross section for emitting a photon of the polarization 1 per volume $\lambda_{c}^{3}$ of the laser is $2.27 \times 10^{-9}\left(\lambda_{c}^{2}\right)$, in which $\lambda_{c}=\hbar / m c=3.86 \times 10^{-13} \mathrm{~m}$ is the Compton wave length of the electron. The last row of table II] shows another example. It is a case of large $\mathcal{N}=523$. The energy of the incident electron is again $3.57 \mathrm{GeV}$, the energy of a photon in the laser is now $4.43 \times 10^{-9} \times 0.51 \mathrm{MeV}=2.259 \mathrm{meV}$, the energy of the emitted photon is $1.9 \times 0.51 \mathrm{MeV}=0.97 \mathrm{MeV}$, and the average differential cross section for emitting a photon of the polarization 1 , per volume $\lambda_{c}^{3}$ of the laser, is $1.08 \times$ $10^{-8}\left(\lambda_{c}^{2}\right)$. The angle $\theta$ between the emitting direction and the direction of the laser propagation is 3.14 for all these cases. From the $k / m$ and $e A / m$ we may calculate the energy flux of the laser. It is $10^{19} \mathrm{~W} / \mathrm{m}^{2}$ for all these seven cases, and is reachable by present techniques.

\section{THE POSSIBILITY OF MAKING A $\gamma$-RAY LASER}

In all cases shown in table I and the case shown in the last row of the table II we see, energies of the emitted photons are in the $\gamma$-ray range. The possibility of constructing a $\gamma$-ray laser by the collision between high energy electrons and an usual laser is worthy to consider. Suppose the electron-laser collision happens in a tube of cross section $S$ and length $L$, with $\sqrt{S} \ll L$. Electrons come from an accelerator, enter the tube at one of its ends, say end 1; then move to another end, say end 2, and exit from the tube there. The laser enters the tube at end 2, then propagates along an opposite direction in the tube to end 1, and exits there. Denote the distance between a point in the tube and the end 1 by $l$. The volume $\mathrm{d} V$ of a space in the tube between two sections at $l$ and $l+\mathrm{d} l$ respectively is $S \mathrm{~d} l$. The total average differential cross section of the laser in this space for an electron of energy $E$ emitting a photon of polarization $i$ and wave vector $\boldsymbol{k}^{\prime}$ is $S \overline{\frac{\mathrm{d} \Sigma}{\mathrm{d} \Omega_{k^{\prime}}}}(i) \frac{\mathrm{d} l}{\lambda_{c}}$. The probability of emitting a photon of this kind by an electron passing through the space $\mathrm{d} V$ is $\overline{\frac{\mathrm{d} \Sigma}{\mathrm{d} \Omega_{k^{\prime}}}}(i) \frac{\mathrm{d} l}{\lambda_{c}}$. The number of photons of this kind emitted by a burst of $n$ those electrons in the space $\mathrm{d} V$ is

$$
\mathrm{d} N=[N(l)+1] a \frac{\mathrm{d} l}{\lambda_{c}}, \quad \text { with } a=n \frac{\overline{\mathrm{d} \Sigma}}{\mathrm{d} \Omega_{k^{\prime}}}(i) /[N(l)+1]
$$

During the emission, the energy of the electron decreases. Suppose there are some techniques being able to supply the electron energy (pump), and maintain it to be the value $E$, the number $n$ of electrons having energy $E$, and therefore the parameter $a$ in this equation, may be a constant in the whole course. In this case, the solution of equation (125) under the initial condition $N(0)=0$ is

$$
N(l)=\mathrm{e}^{a l / \lambda_{c}}-1 .
$$

For $n \sim 1, \overline{\frac{\mathrm{d} \Sigma}{\mathrm{d} \Omega_{k^{\prime}}}}(i) /[N(l)+1] \sim 10^{-8}$, and $L \sim$ meters, $N(L)$ is almost infinity. This is the $\gamma$-ray amplification by the stimulated emission of radiation.

The pump technique may be a way of accelerating electrons along the tube. Since the change of electron energy distribution due to emissions in the tube may be calculated by the present theory, the way of acceleration for maintaining the original electron energy distribution may 
be designed. Another way of pumping is to open more entrances and exits on the wall along the tube, so that one may substitute the bust of electrons with required energy distribution for the old electron burst, which has been changed due to emissions.

The difficulty of finding transition schemes for amplifying $\gamma$-rays is not serious. Many processes are suitable for this purpose 23. The difficulty of finding a resonator for $\gamma$-ray amplification is truly serious. The tube suggested above, if long enough, may play the role, which was played by the resonator in the usual lasers. It makes us feel hopeful.

\section{DISCUSSIONS}

Since the fine structure constant $\alpha$ is small, the electron-photon interaction is weak, the quantum electrodynamics of a system composed of a few electrons and photons in the vacuum may be handled by perturbation. However, because of a huge number of photons in the laser, the electron-laser interaction may be not weak. The quantum electrodynamics could not be handled by perturbation in general, if a laser is present. The way out of this difficulty is to divide the interaction into two parts. One is the interaction, by which an electron absorbs or emits a photon. This part is still weak, and may be handled by perturbation. Another is the interaction between an electron and a laser. This part may be so strong, that the perturbation method breaks down. In this case, one has to find other reliable methods to handle. We considered the electron-laser collision, in which a photon other than those in the laser is emitted. The electron laser interaction makes a distortion of the electron wave, which is exactly solved for the circularly polarized laser. The emission of photon by the electron is handled by perturbation as usual. It is justified by the smallness of the fine structure constant $\alpha$. The theory is as precise as that for the usual quantum electrodynamics treatment of the emission-absorption processes in vacuum. By use of this formulation we see that there may be some phenomena indeed which were interpreted to be the multi-photon processes [1, 2]. But according to our analysis, they are phenomena originated by the electron wave distortion, induced by the laser field. It is therefore truly non-perturbative.

To realize this procedure, we divided the electromagnetic field into two parts. One is the laser and another is the deviation of the electromagnetic field from it, as did in equations (31) and (32). The laser is a given classical field, and the deviation from it is quantized. The quantization of electromagnetic field around one of its classical solution is a generalization of its usual quantization (around the vacuum), and is equivalent to it. In the canonical quantization, to quantize a classical theory of canonical variables $q_{c}$ and $p_{c}$, one substitutes q-numbers $q$ and $p$ for c-numbers $q_{c}$ and $p_{c}$, and applies the quantization rule $q p-p q=\mathrm{i}$ to them. Now for an arbitrary pair of c-number functions $q_{c}(t)$ and $p_{c}(t)$ of time $t$, we may introduce the variables $Q \equiv q-q_{c}(t)$ and $P \equiv p-p_{c}(t)$ instead of $q$ and $p$, to equivalently describe the system. They satisfy the same quantization rule $Q P-P Q=\mathrm{i}$. The quantization based on new pairs of variables is a generalization of the one based on old pairs, and is equivalent to it. Though this substitution is almost trivial, it does offer something new. Besides its application to the electron laser collision here, we applied it to calculate the residual interaction between nucleons on the basis of a relativistic mean field solution for nuclear matter 24].

The main skill in this work is to find a complete set of orthonormal eigenfunctions for equation (52). It is derived from Wolkow solution[11] of Dirac equation for an electron in a plane electromagnetic wave. The result is used in the quantization of electron field in our case. The same set also offers a general solution of the relativistic quantum mechanics for an electron in a circularly polarized electromagnetic field. It may be used in researches of the electron motion in a laser as well. Among others, it may give tips on how to shorten the electron bursts in the laser. It is important in the techniques for laser acceleration of electrons 25.

Eq. (112) shows the existence of the stimulated emission in electron laser collision. In section VII we considered the possibility of making a $\gamma$-ray laser by use of this process. The result is exciting. It is possible to be realized with an acceptable size.

\section{ACKNOWLEDGMENTS}

The work is supported by the Nature Science Foundation of China with grant number 10875003.

\section{APPENDIX: MAIN STEPS FOR DERIVING EQUATION (71)}

They are: 


$$
\begin{aligned}
& \Xi \equiv\left\{1+\frac{k_{c} R}{2}\left[\alpha_{x} \cos k_{c} z+\alpha_{y} \sin k_{c} z-\mathrm{i}\left(\Sigma_{y} \cos k_{c} z-\Sigma_{x} \sin k_{c} z\right)\right]\right\} \\
& \times\left\{1+\frac{k_{c} R^{\prime}}{2}\left[\alpha_{x} \cos k_{c} z+\alpha_{y} \sin k_{c} z+\mathrm{i}\left(\Sigma_{y} \cos k_{c} z-\Sigma_{x} \sin k_{c} z\right)\right]\right\} \\
& =1+\frac{k_{c}^{2} R R^{\prime}}{2}\left(1-\alpha_{z}\right)+\frac{k_{c}}{2}\left(R+R^{\prime}\right)\left(\alpha_{x} \cos k_{c} z+\alpha_{y} \sin k_{c} z\right) \\
& +\frac{\mathrm{i} k_{c}}{2}\left(R^{\prime}-R\right)\left(\Sigma_{y} \cos k_{c} z-\Sigma_{x} \sin k_{c} z\right), \\
& \Theta \equiv\left\{\mathrm{i}^{-n} \mathrm{~J}_{n}\left(p_{\perp} \rho^{\prime}\right) \mathrm{e}^{\mathrm{i} n \varphi^{\prime}} \mathrm{P}_{+}+\mathrm{i}^{-(n-\sigma)} \mathrm{J}_{n-\sigma}\left(p_{\perp} \rho^{\prime}\right) \mathrm{e}^{\mathrm{i}(n-\sigma) \varphi^{\prime}} \mathrm{P}_{-}\right\} \Xi \\
& \times\left\{\mathrm{i}^{n^{\prime}} \mathrm{J}_{n^{\prime}}\left(p_{\perp}^{\prime} \rho^{\prime \prime}\right) \mathrm{e}^{-\mathrm{i} n^{\prime} \varphi^{\prime \prime}} \mathrm{P}_{+}^{\prime}+\mathrm{i}^{\left(n^{\prime}-\sigma^{\prime}\right)} \mathrm{J}_{n^{\prime}-\sigma^{\prime}}\left(p_{\perp}^{\prime} \rho^{\prime \prime}\right) \mathrm{e}^{-\mathrm{i}\left(n^{\prime}-\sigma^{\prime}\right) \varphi^{\prime \prime}} \mathrm{P}_{-}^{\prime}\right\} \\
& =\left[1+\frac{k_{c}^{2} R R^{\prime}}{2}\left(1-\alpha_{z}\right)\right]\left\{\delta _ { \sigma , \sigma ^ { \prime } } \mathrm { i } ^ { n ^ { \prime } - n } \left[\mathrm{J}_{n}\left(p_{\perp} \rho^{\prime}\right) \mathrm{J}_{n^{\prime}}\left(p_{\perp}^{\prime} \rho^{\prime \prime}\right) \mathrm{e}^{\mathrm{i}\left(n \varphi^{\prime}-n^{\prime} \varphi^{\prime \prime}\right)} \mathrm{P}_{+}\right.\right. \\
& \left.+\mathrm{J}_{n-\sigma}\left(p_{\perp} \rho^{\prime}\right) \mathrm{J}_{n^{\prime}-\sigma}\left(p_{\perp}^{\prime} \rho^{\prime \prime}\right) \mathrm{e}^{\mathrm{i}(n-\sigma) \varphi^{\prime}} \mathrm{e}^{-\mathrm{i}\left(n^{\prime}-\sigma\right) \varphi^{\prime \prime}} \mathrm{P}_{-}\right]+\delta_{\sigma,-\sigma^{\prime}} \mathrm{i}^{n^{\prime}-n+\sigma} \\
& \left.\times\left[\mathrm{J}_{n}\left(p_{\perp} \rho^{\prime}\right) \mathrm{J}_{n^{\prime}+\sigma}\left(p_{\perp}^{\prime} \rho^{\prime \prime}\right) \mathrm{e}^{\mathrm{i} n \varphi^{\prime}} \mathrm{e}^{-\mathrm{i}\left(n^{\prime}+\sigma\right) \varphi^{\prime \prime}} \mathrm{P}_{+}+\mathrm{J}_{n-\sigma}\left(p_{\perp} \rho^{\prime}\right) \mathrm{J}_{n^{\prime}}\left(p_{\perp}^{\prime} \rho^{\prime \prime}\right) \mathrm{e}^{\mathrm{i}(n-\sigma) \varphi^{\prime}} \mathrm{e}^{-\mathrm{i} n^{\prime} \varphi^{\prime \prime}} \mathrm{P}_{-}\right]\right\} \\
& +\frac{1}{2}\left[k_{c}\left(R+R^{\prime}\right)\left(\alpha_{x} \cos k_{c} z+\alpha_{y} \sin k_{c} z\right)+\mathrm{i} k_{c}\left(R^{\prime}-R\right)\left(\Sigma_{y} \cos k_{c} z-\Sigma_{x} \sin k_{c} z\right)\right]\left\{\delta_{\sigma, \sigma^{\prime}} \mathrm{i}^{n^{\prime}-n-\sigma}\right. \\
& \times\left[\mathrm{J}_{n}\left(p_{\perp} \rho^{\prime}\right) \mathrm{J}_{n^{\prime}-\sigma}\left(p_{\perp}^{\prime} \rho^{\prime \prime}\right) \mathrm{e}^{\mathrm{i} n \varphi^{\prime}} \mathrm{e}^{-\mathrm{i}\left(n^{\prime}-\sigma\right) \varphi^{\prime \prime}} \mathrm{P}_{-}-\mathrm{J}_{n-\sigma}\left(p_{\perp} \rho^{\prime}\right) \mathrm{J}_{n^{\prime}}\left(p_{\perp}^{\prime} \rho^{\prime \prime}\right) \mathrm{e}^{\mathrm{i}(n-\sigma) \varphi^{\prime}} \mathrm{e}^{-\mathrm{i} n^{\prime} \varphi^{\prime \prime}} \mathrm{P}_{+}\right] \\
& +\delta_{\sigma,-\sigma^{\prime}} \mathrm{i}^{n^{\prime}-n}\left[\mathrm{~J}_{n}\left(p_{\perp} \rho^{\prime}\right) \mathrm{J}_{n^{\prime}}\left(p_{\perp}^{\prime} \rho^{\prime \prime}\right) \mathrm{e}^{\mathrm{i}\left(n \varphi^{\prime}-n^{\prime} \varphi^{\prime \prime}\right)} \mathrm{P}_{-}\right. \\
& \left.\left.-\mathrm{J}_{n-\sigma}\left(p_{\perp} \rho^{\prime}\right) \mathrm{J}_{n^{\prime}+\sigma}\left(p_{\perp}^{\prime} \rho^{\prime \prime}\right) \mathrm{e}^{\mathrm{i}(n-\sigma) \varphi^{\prime}} \mathrm{e}^{\left.-\mathrm{i}\left(n^{\prime}+\sigma\right) \varphi^{\prime \prime}\right)} \mathrm{P}_{+}\right]\right\} ;
\end{aligned}
$$

and

$$
\int_{0}^{\infty} \int_{0}^{2 \pi} u_{\sigma}^{\dagger}(0) \Theta u_{\sigma^{\prime}}(0) \rho \mathrm{d} \rho \mathrm{d} \varphi=\frac{\pi}{p_{\perp}} \delta\left(p_{\perp}-p_{\perp}^{\prime}\right) \mathrm{e}^{\mathrm{i}\left[\frac{\sigma^{\prime}-\sigma}{2}+n-n^{\prime}\right] k_{c} z} W
$$

with

$$
\begin{aligned}
& W \equiv u_{\sigma}^{\dagger}(0)\left\{\left[2+k_{c}^{2} R R^{\prime}\left(1-\alpha_{z}\right)\right]\left[\delta_{\sigma, \sigma^{\prime}} \mathrm{J}_{n-n^{\prime}}\left(p_{\perp} \Delta R\right)+\delta_{\sigma,-\sigma^{\prime}} \mathrm{J}_{n-n^{\prime}-\sigma}\left(p_{\perp} \Delta R\right)\right]\right. \\
& -k_{c}\left[\left(\Sigma R \gamma_{5}+\Delta R\right) \Sigma_{-}+\left(\Sigma R \gamma_{5}-\Delta R\right) \Sigma_{+}\right]\left[\delta_{\sigma, \sigma^{\prime}}\left(\mathrm{J}_{n-n^{\prime}+\sigma}\left(p_{\perp} \Delta R\right) \mathrm{P}_{-}+\mathrm{J}_{n-n^{\prime}-\sigma}\left(p_{\perp} \Delta R\right) \mathrm{P}_{+}\right)\right. \\
& \left.\left.+\delta_{\sigma,-\sigma^{\prime}}\left(\mathrm{J}_{n-n^{\prime}}\left(p_{\perp} \Delta R\right) \mathrm{P}_{-}+\mathrm{J}_{n-n^{\prime}-2 \sigma}\left(p_{\perp} \Delta R\right) \mathrm{P}_{+}\right)\right]\right\} u_{\sigma^{\prime}}(0),
\end{aligned}
$$

in which $\Sigma R \equiv R+R^{\prime}, \Delta R \equiv R^{\prime}-R, \Sigma_{ \pm} \equiv \frac{\Sigma_{x} \pm \mathrm{i} \Sigma_{y}}{2}, \gamma_{5} \equiv$ $\gamma_{1} \gamma_{2} \gamma_{3} \gamma_{4}=-\alpha_{i} \Sigma_{i}, i=x, y, z$. The formula (70), formulae

$$
\left.\begin{array}{l}
\alpha_{i} \alpha_{j}+\alpha_{j} \alpha_{i}=2 \delta_{i, j}, \\
\Sigma_{i} \Sigma_{j}+\Sigma_{j} \Sigma_{i}=2 \delta_{i, j}
\end{array}\right\} \quad \text { for } i, j=x, y, z
$$

the orthogonal relations

$$
\left.\begin{array}{l}
\int_{0}^{\infty} \mathrm{J}_{n}\left(p_{\perp} \rho\right) \mathrm{J}_{n}\left(p_{\perp}^{\prime} \rho\right) \rho \mathrm{d} \rho=\frac{1}{p_{\perp}} \delta\left(p_{\perp}-p_{\perp}^{\prime}\right), \\
\int_{0}^{2 \pi} \mathrm{e}^{\mathrm{i}\left(\nu-\nu^{\prime}\right) \varphi} \mathrm{d} \varphi=2 \pi \delta_{\nu, \nu^{\prime}},
\end{array}\right\}
$$

and the addition theorem

$$
\sum_{n^{\prime}=-\infty}^{\infty} \mathrm{J}_{n+n^{\prime}}\left(\xi_{1}\right) \mathrm{J}_{n^{\prime}}\left(\xi_{2}\right)=\mathrm{J}_{n}\left(\xi_{1}-\xi_{2}\right)
$$

are used. Now,

$$
I=\frac{\pi}{p_{\perp}} \delta\left(p_{\perp}-p_{\perp}^{\prime}\right) \int_{-\infty}^{\infty} \mathrm{e}^{\mathrm{i}\left(\eta_{n^{\prime}}-\eta_{n}\right) z} W \mathrm{~d} z
$$

with $\eta_{n}=p_{z}+\frac{e^{2} A_{c}^{2}}{2\left(E-p_{z}\right)}+\left(\frac{\sigma}{2}-n\right) k_{c}$ and $\eta_{n^{\prime}}=p_{z}^{\prime}+$ $\frac{e^{2} A_{c}^{2}}{2\left(E^{\prime}-p_{z}^{\prime}\right)}+\left(\frac{\sigma^{\prime}}{2}-n^{\prime}\right) k_{c}$. Since $W$ is $z$ independent, the nonzero condition for the integral in eq. (134) is $\eta_{n}=\eta_{n^{\prime}}$. The nonzero conditions for $I$ include $\varepsilon_{n}=\varepsilon_{n^{\prime}}, \eta_{n}=\eta_{n^{\prime}}$ and $p_{\perp}=p_{\perp}^{\prime}$. After a careful analysis we see that they mean $p_{\perp}=p_{\perp}^{\prime}, p_{z}=p_{z}^{\prime}, \tau=\tau^{\prime}, \Delta R=0, n=n^{\prime}$ if $\sigma=\sigma^{\prime}, n^{\prime}=n-\sigma$ if $\sigma=-\sigma^{\prime}$. Since $\mathrm{J}_{n}(0)=\delta_{n, 0}$ and $u_{\sigma}^{\dagger}(0) \alpha_{z} u_{\sigma^{\prime}}(0)=\frac{p_{z}}{E} \delta_{\sigma, \sigma^{\prime}}$, we finally obtain 


$$
\begin{aligned}
I & =\frac{4 \pi^{2}}{p_{\perp}}\left[1+\frac{k^{2} R^{2}}{2}\left(1-\frac{p_{z}}{E}\right)\right] \delta\left(p_{\perp}-p_{\perp}^{\prime}\right) \delta\left(\eta-\eta^{\prime}\right) \delta_{n, n^{\prime}} \delta_{\sigma, \sigma^{\prime}} \delta_{\tau, \tau^{\prime}} \\
& =\frac{4 \pi^{2}}{p_{\perp}}\left[1+\frac{k^{2} R^{2}}{2}\left(1-\frac{p_{z}}{E}\right)\right] \frac{2 E\left(E-p_{z}\right)}{2 E\left(E-p_{z}\right)+e^{2} A_{c}^{2}} \delta\left(p_{\perp}-p_{\perp}^{\prime}\right) \delta\left(p_{z}-p_{z}^{\prime}\right) \delta_{n, n^{\prime}} \delta_{\sigma, \sigma^{\prime}} \delta_{\tau, \tau^{\prime}} \\
& =\frac{4 \pi^{2}}{p_{\perp}} \delta\left(p_{\perp}-p_{\perp}^{\prime}\right) \delta\left(p_{z}-p_{z}^{\prime}\right) \delta_{n, n^{\prime}} \delta_{\sigma, \sigma^{\prime}} \delta_{\tau, \tau^{\prime}} .
\end{aligned}
$$

[1] Voronov G S and Delone N B 1966 Sov. Phys. JETP 23 54

[2] Agostini P,Fabre F,Mainfray G, Petite G and Rahman N K 1979 Phys. Rev. Lett. 42, 1127

[3] Bucksbaum P H, Schumacher D W and Bashkansky M 1988 Phys. Rev. Lett. 611182

[4] Guo D S, Freeman R R and Wu Y S 2000 J. Phys. A33 7955

[5] Zhang Q R 1996 Phys. Lett. A 216125

[6] Ledingham W D, McKenna P and Singhal R P 2003 Science $\mathbf{3 0 0} 1107$

[7] Ao S Y, Cheng T W, Li X F, Pan S F and Fu P M 2003 Chin. Phys. Lett. 20499

[8] Zhang Q R 2007 Commun. Theor. Phys. 471017

[9] Xie B S, Mohamedsedik M and Dulat S 2012 Chin. Phys. Lett. 29021102

[10] Ren N, Wang J X, Li A K and Wang P X 2012 Chin. Phys. Lett. 29071201

[11] Wolkow D M 1935 Z. Physik 94250

[12] Zhang Q R 2002 Quantum Mechanics(in chinese) (Beijing: Science Press )
[13] Weinberg S 1995 The Quantum Theory of Fields I ( Cambridge: Cambridge Press)

[14] Lurie D 1968 Particles and Fields ( New York: Interscience Publisher)

[15] Watson G N 1958 A treatise on the theory of Bessel Functions (Cambridge: Cambridge University Press )

[16] Wang Z X and Guo D R 1965 Theory of Special Functions(in Chinese) (Beijing: Science Press)

[17] Gell-Mann M and Low F 1951 Phys. Rev. 84350

[18] Compton A H 1923 Phys. Rev. 21483

[19] Compton A H 1923 Phys. Rev. 22409

[20] Klein O and Nishina Y. 1929 Z. Physik 52853

[21] Nishina Y. 1929 Z. Physik 52869

[22] Milburn R H 1963 Phys. Rev. Lett. 1075

[23] Zhang Q R , Gao C Y and Yao S D 2001 Chin. Scien. Bull. 461238

[24] Zhang Q R and Gao C Y 2011 Commun. Theor. Phys. $\mathbf{5 5} 765$

[25] Christopher M S S , Colby E R, Cowan B M, Siemann R H and Spencer J E 2005 Phys. Rev. Lett. 95, 194801 